\title{
ON THE CLASSIFICATION AND BIFURCATION OF MULTIGERMS OF MAPS FROM SURFACES TO 3-SPACE
}

\author{
C. A. HOBBS and N. P. KIRK*
}

\begin{abstract}
The $\mathscr{A}$-classification of multigerm singularities is discussed, based on the theory of complete transversals. An $\mathscr{A}$-classification of $r$-multigerms from the plane to 3 -space of $\mathscr{A}$-codimension $\leq$ $6-r$ is carried out and the bifurcation geometry of these singularities analysed. This work has applications to the study of two-dimensional spatial motions, giving local models for the singularities which appear on general trajectories of rigid body motions from the plane to 3space. In addition, our classification is extensive enough to give the full list of simple multigerm singularities from the plane to 3 -space.
\end{abstract}

\section{Introduction}

In theoretical singularity theory one usually concentrates on strictly local phenomena (monogerms). However, in applications there is often no reason to restrict to such cases, it is just as important to consider the semi-local singularities (multigerms). We consider two main objectives in this paper. Firstly to give an account of how certain techniques from singularity theory apply in the case of multigerms. Although multigerms have been considered in previous articles there are few works which have them as their central concern. We show how various familiar techniques for monogerms carry over to concrete examples. In addition we describe new, efficient and general techniques for the classification of multigerms. Specifically, we describe how the method of Complete Transversals developed in the recent article by Bruce, du Plessis and the second author [2] may be applied to the case of multigerm classification. This work demonstrated that typical calculations required in the classification process may be carried out using a computer and subsequently a specialist classification package called Transversal was written by the second author [9]. The present work involved writing and using an extension of this package which deals with multigerms.

\footnotetext{
* The second author was supported by the EPSRC grants GR/J28162, GR/L33948 and GR/L17245; computational work was supported by grant GR/H59855.

Received June 15, 1998
} 
Our second objective is to apply these techniques to an important case: the classification and bifurcation of multigerm singularities $\mathrm{R}^{2}, S \rightarrow \mathrm{R}^{3}, 0$. Although some listing has been undertaken by Mond [12] and Goryunov [7] ours is the first systematic classification of multigerm singularities. Our aim is to give a comprehensive and extensive list which is suitable as a reference for future applications. We also give a detailed description of the bifurcation geometry which, in the case of semi-local surface singularities in three-space, is rich and can be presented graphically. A comprehensive series of pictures, analysed with the help of a specialist computer graphics package, is given.

The following problem from theoretical kinematics provided our primary motivation: describe the geometry associated to singularities of trajectories for general rigid body motions of the plane and space, with various degrees of freedom. Some work has been carried out in this area by Gibson and the first author. In particular [4] in which a theorem relating classical singularity theory to this problem is proved; [5] in which this theory is applied to planar motions; and [6] which describes the geometry associated with singularities of trajectories in 3-space with 2 degrees of freedom and a single branch. The main theorem from [4] enables us to decide which singularities will appear generically on trajectories of rigid body motions by giving restrictions on the possible codimension of such singularities. The results from this paper, together with those for the monogerm case [6], give us the local models describing the geometry of trajectories of rigid body motions with 2 degrees of freedom in 3-space, thus providing a mathematical foundation for future analysis.

Classifications of the type presented in this paper are of importance in singularity theory also. For example, it is natural to ask how Mond's topological study of $\mathscr{A}$-finite monogerms [13] extends to the multigerm case. To extend the work of Goryunov [7] on local invariants of mappings of surfaces into threespace to higher order invariants, one needs a classification of multigerms up to a corresponding higher codimension. A classification list such as ours thus provides basic results which are needed for a variety of future avenues of research.

Our list of singularities based on codimension appears in Table 2. Further calculations gave us the full list of simple singularities; see Table 1. These represent our main results on multigerm classification and are summarised in Theorems 1.2 and 1.1 following. The classification techniques are described in Section 2; Section 3 provides the details of the classification and notation used; finally Section 4 describes the bifurcation geometry.

Some of the results in this paper appeared in the first author's Ph.D. thesis [8]. The results are verified and then considerably extended in this paper using the computer classification package Transversal. Since writing this paper it has been brought to our attention that a similar project is currently under in- 
TABLE 1. Simple Multigerms $\mathrm{R}^{2}, S \rightarrow \mathrm{R}^{3}, 0$.

\begin{tabular}{|l|l|c|c|l|}
\hline Name & Normal Form & Det & $\mathscr{A}_{e}$-cod & Ref \\
\hline$A_{0}$ (immersion) & $(x, y, 0)$ & 1 & 0 & - \\
$S_{0}$ (cross-cap) & $\left(x, y^{2}, x y\right)$ & 2 & 0 & - \\
$S_{k}^{ \pm}$ & $\left(x, y^{2}, y^{3} \pm x^{k+1} y\right)$ & $k+2$ & $k$ & - \\
$B_{k}^{ \pm}$ & $\left(x, y^{2}, x^{2} y \pm y^{2 k+1}\right), k \geq 2$ & $2 k+1$ & $k$ & - \\
$C_{k}^{ \pm}$ & $\left(x, y^{2}, x y^{3} \pm x^{k} y\right), k \geq 3$ & $k+1$ & $k$ & - \\
$F_{4}$ & $\left(x, y^{2}, x^{3} y+y^{5}\right)$ & 5 & 4 & - \\
$H_{k}$ & $\left(x, x y+y^{3 k-1}, y^{3}\right), k \geq 2$ & $3 k-1$ & $k$ & - \\
\hline$A_{0}^{2}$ & $(x, y, 0 ; 0, X, Y)$ & 1 & 0 & 1 \\
$A_{0}^{2} \mid A_{k}^{ \pm}$ & $\left(x, y, 0 ; X, Y, X^{2} \pm Y^{k+1}\right)$ & $k+1$ & $k$ & 2 \\
$A_{0}^{2} \mid D_{k}^{ \pm}$ & $\left(x, y, 0 ; X, Y, X^{2} Y \pm Y^{k-1}\right), k \geq 4$ & $k-1$ & $k$ & 2 \\
$A_{0}^{2} \mid E_{6}$ & $\left(x, y, 0 ; X, Y, X^{3}+Y^{4}\right)$ & 4 & 6 & 2 \\
$A_{0}^{2} \mid E_{7}$ & $\left(x, y, 0 ; X, Y, X^{3}+X Y^{3}\right)$ & 4 & 7 & 2 \\
$A_{0}^{2} \mid E_{8}$ & $\left(x, y, 0 ; X, Y, X^{3}+Y^{5}\right)$ & 5 & 8 & 2 \\
$\left(A_{0} S_{0}\right)_{k}$ & $\left(x, y, 0 ; Y^{2}, X Y+Y^{2 k+1}, X\right)$ & $2 k+1$ & $k$ & 3.1 \\
$A_{0} S_{k}^{ \pm}$ & $\left(x, y, 0 ; Y^{3} \pm X^{k+1} Y, Y^{2}, X\right)$ & $k+2$ & $k+1$ & $3.2 .1 / 2$ \\
$A_{0} S_{0} \mid A_{k}^{ \pm}$ & $\left(x, y, 0 ; X, X Y, Y^{2} \pm X^{k+1}\right)$ & $k+1$ & $k+1$ & 4 \\
$\left(A_{0} S_{0} \mid A_{\infty}\right)_{k}$ & $\left(x, y, 0 ; X, Y^{2}, X Y+Y^{2 k}\right), k \geq 2$ & $2 k$ & $k+1$ & 4 \\
\hline$A_{0}^{3}$ & $(x, y, 0 ; X, 0, Y ; 0, \bar{x}, \bar{y})$ & 1 & 0 & 1 \\
$A_{0}^{3} \mid A_{k}$ & $(x, y, 0 ; X, 0, Y ; \bar{x}, \bar{y}, \bar{y}+\bar{x} k+1)$ & $k+1$ & $k$ & 2 \\
$\left(A_{0}^{2} \mid A_{k}^{ \pm}\right)\left(A_{0}\right)$ & $\left(x, y, 0 ; X, 0, Y ; \bar{x}, \bar{y}, \bar{x}^{2} \pm \bar{y} k+1\right)$ & $k+1$ & $k+1$ & $3.1 / 2$ \\
$\left(A_{0}^{2} \mid A_{\infty}\right)\left(A_{0}\right) \mid A_{k}$ & $(x, y, 0 ; X, 0, Y ; \bar{x}, \bar{y}, \bar{x} \bar{y}+\bar{x} k+1), k \geq 2$ & $k+1$ & $k+1$ & 3.3 \\
$\left(A_{0}^{2} \mid A_{2}\right)\left(A_{0}\right) \mid A_{2}$ & $\left(x, y, 0 ; X, 0, Y ; \bar{x}, \bar{y}, \bar{y}^{2}+\bar{x}^{3}\right)$ & 3 & 3.4 \\
\hline$A_{0}^{4}$ & $(x, y, 0 ; X, 0, Y ; 0, \bar{x}, \bar{y} ; \bar{X}, \bar{Y}, \bar{Y}+\bar{X})$ & 1 & 1 \\
$\left(A_{0}^{3} \mid A_{k}\right)\left(A_{0}\right)$ & $\left(x, y, 0 ; X, 0, Y ; 0, \bar{x}, \bar{y} ; \bar{X}, \bar{Y}, \bar{Y}+\bar{X}^{k+1}\right)$ & $k+1$ & $k+1$ & 2 \\
\hline & & & & \\
\hline
\end{tabular}

$k \geq 1$ unless otherwise stated.

vestigation by Atique, Mond and Ruas [1]. However, their main concern is with the simple singularities and they use a completely different classification scheme, more suited to identifying series rather than a complete stratification of the jet-space with respect to codimension. In classifications of this nature we cannot hope to describe all of the details, indeed Section 3 is mainly a summary of our findings. It is therefore worth pointing out that parts of our list have been reproduced and verified using different techniques in the aforementioned work. The two projects therefore serve as a useful check on their common results. They only overlap in parts of the classification and here our work predates theirs; the other parts of the two articles address different applications. 
TABLE 2. $r$-multigerms satisfying $\mathscr{A}$-codimension(strata) $\leq 6-r$.

\begin{tabular}{|c|c|c|c|c|}
\hline Name & Normal Form & Det & $\mathscr{A}_{e}$-cod & Ref \\
\hline$A_{0}^{2}$ & $(x, y, 0 ; 0, X, Y)$ & 1 & 0 & 1 \\
\hline$A_{0}^{2} \mid A_{1}^{ \pm}$ & $\left(x, y, 0 ; X, Y, X^{2} \pm Y^{2}\right)$ & 2 & 2 & 2 \\
\hline$A_{0}^{2} \mid A_{2}$ & $\left(x, y, 0 ; X, Y, X^{2}+Y^{3}\right)$ & 3 & 3 & 2 \\
\hline$A_{0}^{2} \mid A_{3}^{ \pm}$ & $\left(x, y, 0 ; X, Y, X^{2} \pm Y^{4}\right)$ & 4 & 4 & 2 \\
\hline$\left(A_{0} S_{0}\right)_{1}$ & $\left(x, y, 0 ; Y^{2}, X Y+Y^{3}, X\right)$ & 3 & 2 & 3.1 \\
\hline$\left(A_{0} S_{0}\right)_{2}$ & $\left(x, y, 0 ; Y^{2}, X Y+Y^{5}, X\right)$ & 5 & 3 & 3.1 \\
\hline$\left(A_{0} S_{0}\right)_{3}$ & $\left(x, y, 0 ; Y^{2}, X Y+Y^{7}, X\right)$ & 7 & 4 & 3.1 \\
\hline$A_{0} S_{1}^{ \pm}$ & $\left(x, y, 0 ; Y^{3} \pm X^{2} Y, Y^{2}, X\right)$ & 3 & 3 & 3.2 .1 \\
\hline$A_{0} S_{2}$ & $\left(x, y, 0 ; Y^{3}+X^{3} Y, Y^{2}, X\right)$ & 4 & 4 & 3.2 .2 \\
\hline$A_{0} B_{2}$ & $\left(x, y, 0 ; X^{2} Y+X Y^{3}+a Y^{5}, Y^{2}, X\right)$ & $5, a \neq 0, \frac{1}{4}$ & 5 & 3.2 .3 \\
\hline $\mathrm{A}_{0} \mathrm{H}_{2}$ & $\left(x, y, 0 ; Y^{3}+Y^{4}, X Y+Y^{4}+a Y^{5}, X\right)$ & $5, a \neq 1$ & 5 & 3.3 \\
\hline$A_{0} S_{0} \mid A_{1}^{ \pm}$ & $\left(x, y, 0 ; X, X Y, Y^{2} \pm X^{2}\right)$ & 2 & 3 & 4 \\
\hline$A_{0} S_{0} \mid A_{2}$ & $\left(x, y, 0 ; X, X Y, Y^{2}+X^{3}\right)$ & 3 & 4 & 4 \\
\hline$A_{0} S_{1} \mid A_{1}^{ \pm}$ & $\left(x, y, 0 ; X, Y^{3}+a X^{2} Y, Y^{2} \pm X^{2}\right)$ & $3, a \neq 0, \pm 1^{\dagger}$ & 5 & 4 \\
\hline$\left(A_{0} S_{0} \mid A_{\infty}\right)_{2}$ & $\left(x, y, 0 ; X, Y^{2}, X Y+Y^{4}\right)$ & 4 & 4 & 4 \\
\hline$S_{0} S_{0}$ & $\left(x, x y+y^{3}, y^{2} ; X Y+a Y^{3}, X, Y^{2}\right)$ & $3, a \neq 0$ & 5 & 5 \\
\hline$A_{0}^{3}$ & $(x, y, 0 ; X, 0, Y ; 0, \bar{x}, \bar{y})$ & 1 & 0 & 1 \\
\hline$A_{0}^{3} \mid A_{1}$ & $\left(x, y, 0 ; X, 0, Y ; \bar{x}, \bar{y}, \bar{y}+\bar{x}^{2}\right)$ & 2 & 1 & 2 \\
\hline$A_{0}^{3} \mid A_{2}$ & $\left(x, y, 0 ; X, 0, Y ; \bar{x}, \bar{y}, \bar{y}+\bar{x}^{3}\right)$ & 3 & 2 & 2 \\
\hline$A_{0}^{3} \mid A_{3}$ & $\left(x, y, 0 ; X, 0, Y ; \bar{x}, \bar{y}, \bar{y}+\bar{x}^{4}\right)$ & 4 & 3 & 2 \\
\hline$\left(A_{0}^{2} \mid A_{1}^{ \pm}\right)\left(A_{0}\right)$ & $\left(x, y, 0 ; X, 0, Y ; \bar{x}, \bar{y}, \bar{x}^{2} \pm \bar{y}^{2}\right)$ & 2 & 2 & 3.1 \\
\hline$\left(A_{0}^{2} \mid A_{2}\right)\left(A_{0}\right)$ & $\left(x, y, 0 ; X, 0, Y ; \bar{x}, \bar{y}, \bar{x}^{2}+\bar{y}^{3}\right)$ & 3 & 3 & 3.2 \\
\hline$\left(A_{0}^{2} \mid A_{\infty}\right)\left(A_{0}\right) \mid A_{2}$ & $\left(x, y, 0 ; X, 0, Y ; \bar{x}, \bar{y}, \bar{x} \bar{y}+\bar{x}^{3}\right)$ & 3 & 3 & 3.3 \\
\hline$A_{0}^{2} S_{0} \mid A_{1}$ & $\left(x, y, 0 ; X, 0, Y ; \bar{x} \bar{y}+a \bar{y}^{3}, \bar{x}, \bar{x}+\bar{y}^{2}\right)$ & $3, a \neq 0,1$ & 3 & 4 \\
\hline$A_{0}^{2} S_{0} \mid A_{1}(0)^{ \pm}$ & $\left(x, y, 0 ; X, 0, Y ; \bar{x} \bar{y} \pm \bar{y}^{5}, \bar{x}, \bar{x}+\bar{y}^{2}\right)$ & 5 & 3 & 4 \\
\hline$A_{0}^{2} S_{0} \mid A_{1}(1)^{ \pm}$ & $\left(x, y, 0 ; X, 0, Y ; \bar{x} \bar{y}+\bar{y}^{3} \pm \bar{y}^{5}, \bar{x}, \bar{x}+\bar{y}^{2}\right)$ & 5 & 3 & 4 \\
\hline$A_{0}^{2} S_{1}^{ \pm} \mid A_{1}$ & $\left(x, y, 0 ; X, 0, Y ; \bar{y}^{3} \pm \bar{x}^{2} \bar{y}, \bar{x}, \bar{x}+\bar{y}^{2}\right)$ & 3 & 3 & 4 \\
\hline$A_{0}^{2} S_{0} \mid A_{2}$ & $\left(x, y, 0 ; X, 0, Y ; \bar{y}^{2}, \bar{x}, \bar{x}+\bar{x} \bar{y}+\bar{y}^{3}\right)$ & 3 & 3 & 4 \\
\hline$\left(A_{0} S_{0} \mid A_{1}^{ \pm}\right)\left(A_{0}\right)_{1}$ & $\left(x, y, 0 ; X, 0, Y ; \bar{x} \bar{y}+\bar{y}^{3}, \bar{x}, \bar{y}^{2} \pm \bar{x}^{2}\right)$ & 3 & 3 & 5 \\
\hline$A_{0}^{4}$ & $(x, y, 0 ; X, 0, Y ; 0, \bar{x}, \bar{y} ; \bar{X}, \bar{Y}, \bar{Y}+\bar{X})$ & 1 & 0 & 1 \\
\hline$\left(A_{0}^{3} \mid A_{1}\right)\left(A_{0}\right)$ & $\left(x, y, 0 ; X, 0, Y ; 0, \bar{x}, \bar{y} ; \bar{X}, \bar{Y}, \bar{Y}+\bar{X}^{2}\right)$ & 2 & 1 & 2 \\
\hline$\left(A_{0}^{3} \mid A_{2}\right)\left(A_{0}\right)$ & $\left(x, y, 0 ; X, 0, Y ; 0, \bar{x}, \bar{y} ; \bar{X}, \bar{Y}, \bar{Y}+\bar{X}^{3}\right)$ & 3 & 2 & 2 \\
\hline$\left(A_{0}^{2} \mid A_{1}\right)\left(A_{0}^{2}\right)$ & $\begin{array}{l}(x, y, 0 ; X, 0, Y ; 0, \bar{x}, \bar{y} \\
\left.\quad \bar{X}, \bar{Y}, \bar{Y}^{2}+\bar{X} \bar{Y}+a \bar{X}^{2}\right)\end{array}$ & $2, a \neq 0, \frac{1}{4}$ & 3 & 3 \\
\hline
\end{tabular}


TABLE 2. continued

\begin{tabular}{|c|c|c|c|c|}
\hline $\begin{array}{l}A_{0}^{5} \\
\left(A_{0}^{3} \mid A_{1}\right)\left(A_{0}^{2}\right)\end{array}$ & $\begin{array}{l}(x, y, 0 ; X, 0, Y ; 0, \bar{x}, \bar{y} ; \bar{X}, \bar{Y}, \bar{X}+\bar{Y} \\
\quad \tilde{x}, \tilde{y}, a \tilde{x}+b \tilde{y}) \\
\left(x, y, 0 ; X, 0, Y ; 0, \bar{x}, \bar{y} ; \bar{X}, \bar{Y}, \bar{Y}+\bar{X}^{2}\right. \\
\quad \tilde{x}, \tilde{y}, \tilde{x}+a \tilde{y})\end{array}$ & $\begin{array}{c}1^{\ddagger} \\
2, a \neq 0,1\end{array}$ & 2 & 1 \\
\hline$A_{0}^{6}$ & $\begin{array}{l}\left(x, y, 0 ; X, 0, Y ; \bar{x}^{2}, \bar{x}, \bar{y} ; \bar{X}, \bar{Y}, \bar{X}+\bar{Y}\right. \\
\quad \tilde{x}, \tilde{y}, a_{1} \tilde{x}+a_{2} \tilde{y}+a_{3} \tilde{x}^{2} \\
\left.\quad \tilde{X}, \tilde{Y}, a_{4} \tilde{X}+a_{5} \tilde{Y}+a_{6} \tilde{X}^{2}\right)\end{array}$ & $\dagger$ & 6 & - \\
\hline
\end{tabular}

${ }^{\dagger}$ Here $a \neq \pm 1$ is respective of the sign of the $X^{2}$ coefficient.

$¥$-determined provided $a, b, a-1, b-1, a-b \neq 0$.

$\dagger$ finitely-determined for generic values of the $a_{i}$; see Section 3.5.

TheOREm 1.1. Table 1 lists, up to $\mathscr{A}$-equivalence, all simple multigerms $\mathrm{R}^{2}, S \rightarrow \mathrm{R}^{3}, 0$ of multiplicity greater than 1 . The simple monogerms were classified by Mond in [12], these are also shown.

THEOREM 1.2. Table 2 lists all multigerms $\mathrm{R}^{2}, S \rightarrow \mathrm{R}^{3}, 0$ (and multigerm strata where moduli feature) which can be local models for the singularities appearing on the trajectories of rigid body motions from the plane to space. Explicitly, we list all $r$-multigerms satisfying $\mathscr{A}$-codimension(strata) $\leq 6-r$. The table is divided into sections according to the multiplicity $r=2, \ldots, 6$ of the multigerm. The column marked 'Det' gives the determinacy degree and restrictions on any moduli. Moduli are denoted by $a, b$ or $a_{i}$ and when moduli feature the $\mathscr{A}$-codimension refers to the $\mathscr{A}$-codimension of a member of the stratum. (For the classification of monogerms we refer the reader to Mond's Table 1, p.337 in [12]. Those monogerms relevant to the kinematics problem were analysed in [6].)

The column labelled 'Ref' in Tables 1 and 2 references the 'case number' which deals with the multigerm in Sections 3.1 to 3.5. The \pm signs appearing in the normal forms in Table 2 represent distinct types. In Table 1 the notation is used as a convenient method of representing the whole series: here the \pm signs represent distinct types depending on the parity of the exponent $k$.

Acknowledgements. We would like to thank Professor J. W. Bruce and Dr. C. G. Gibson for helpful discussions and Dr. R. J. Morris whose computer package LSMP, The Liverpool Surface Modelling Package [14], was used to investigate the unfolding spaces in Section 4. We would also like to thank the referee for making several useful suggestions which we have incorporated. The second author was supported by the EPSRC grants GR/J28162, GR/L33948 and GR/L17245; computational work was supported by grant GR/H59855. 


\section{Multigerms: Preliminaries and Techniques}

We refer to the survey article of Wall [15], for background singularity theory. A few further remarks concerning multigerms will be helpful. Given $S \subset \mathrm{R}^{n}$ and $T \subset \mathrm{R}^{p}$, a multigerm $f: \mathrm{R}^{n}, S \rightarrow \mathrm{R}^{p}, T$ will mean an equivalence class of $C^{\infty}$ mappings $\tilde{f}: U \rightarrow \mathrm{R}^{p}$, where $U$ is an open neighbourhood of $S$ in $\mathrm{R}^{n}$ and $\tilde{f}(S) \subset T$, with two representatives deemed to be equivalent if they agree on an open neighbourhood of $S$. In the special case $S=\{0\} \subset \mathrm{R}^{n}$, $T=\{0\} \subset \mathrm{R}^{p}$ we call such germs monogerms. The notion of $\mathscr{A}$-equivalence extends to multigerms in the obvious way.

The ring of function germs $f: \mathrm{R}^{n}, 0 \rightarrow \mathrm{R}$ is denoted $\mathscr{E}_{n}$; this is a local ring with maximal ideal $\mathscr{M}_{n}$ consisting of those germs $f$ with $f(0)=0$. The $\mathscr{E}_{n}$-module of monogerms $f: \mathrm{R}^{n}, 0 \rightarrow \mathrm{R}^{p}$ is denoted $\mathscr{E}(n, p)$; the submodule consisting of those germs $f$ with $f(0)=0$ is given by $\mathscr{M}_{n} \mathscr{E}(n, p)$.

We will be interested in multigerms for which $S$ is of finite cardinality, $S=$ $\left\{x_{1}, \ldots, x_{r}\right\}$ say, and $T=\{0\}$. The set of all multigerms $f: \mathrm{R}^{n}, S \rightarrow \mathrm{R}^{p}, 0$ is then isomorphic to the direct sum of $r$ copies of $\mathscr{M}_{n} \mathscr{E}(n, p)$. This realisation is far easier to work with and from now on such $r$-multigerms will be denoted $\left(f_{1} ; \ldots ; f_{r}\right)$, where $f_{i}: \mathrm{R}^{n}, 0 \rightarrow \mathrm{R}^{p}, 0$. (Note that $\oplus_{1}^{r} \mathscr{M}_{n} \mathscr{E}(n, p)$ is a finitely generated $\oplus_{1}^{r} \mathscr{E}_{n}$-module, where scalar multiplication is given by $\left(\lambda_{1} ; \ldots ; \lambda_{r}\right)$. $\left.\left(f_{1} ; \ldots ; f_{r}\right)=\left(\lambda_{1} f_{1} ; \ldots ; \lambda_{r} f_{r}\right).\right)$ The integer $r$ is called the multiplicity of the multigerm, and the $f_{i}$ its branches. In this notation $\mathscr{A}$-equivalence of multigerms can be defined as follows: $\left(f_{1} ; \ldots ; f_{r}\right) \sim_{\mathscr{A}}\left(g_{1} ; \ldots ; g_{r}\right)$ if there exists germs of diffeomorphisms $h_{1}, \ldots, h_{r}: \mathrm{R}^{n}, 0 \rightarrow \mathrm{R}^{n}, 0$ and $H: \mathrm{R}^{p}, 0 \rightarrow$ $\mathrm{R}^{p}, 0$ such that

$$
\left(f_{1} ; \ldots ; f_{r}\right)=\left(H \circ g_{1} \circ h_{1} ; \ldots ; H \circ g_{r} \circ h_{r}\right) .
$$

As in the monogerm case, this equivalence may be induced by the natural action of the group $\mathscr{A}=\left(\oplus_{1}^{r} \mathscr{R}\right) \oplus \mathscr{L}$.

REMARK 1. (i) In the source we are free to change coordinates about each base point independently of the associated branch, whereas in the target the same coordinate change must be applied to each branch.

(ii) Strictly speaking the order of branches is irrelevant so we are really working with the quotient of $\oplus_{1}^{r} \mathscr{M}_{n} \mathscr{E}(n, p)$ under the natural action of the permutation group $S(r)$ as well as that of $\mathscr{A}$.

Let $\left(x_{1}, \ldots, x_{n}\right)$ denote coordinates on $\mathrm{R}^{n} ;\left\{e_{1}, \ldots, e_{p}\right\}$ denote the standard basis vectors on $\mathrm{R}^{p}$ (considered as monogerms $\mathrm{R}^{n}, 0 \rightarrow \mathrm{R}^{p}$ ); and let $f$ be the multigerm $\left(f_{1} ; \ldots ; f_{r}\right)$. The tangent space to the orbit $\mathscr{A} \cdot f$ is given via the standard technique of considering paths in $\mathscr{A}$ through the identity. We find 
that, in coordinate form, $L \mathscr{A} \cdot f=L \mathscr{R} \cdot f+L \mathscr{L} \cdot f$ where

$$
\begin{aligned}
L \mathscr{R} \cdot f & =\mathscr{M}_{n}\left(\frac{\partial f_{1}}{\partial x_{1}}, \ldots, \frac{\partial f_{1}}{\partial x_{n}}\right) \oplus \cdots \oplus \mathscr{M}_{n}\left(\frac{\partial f_{r}}{\partial x_{1}}, \ldots, \frac{\partial f_{r}}{\partial x_{n}}\right), \\
L \mathscr{L} \cdot f & =\left(f_{1} ; \ldots ; f_{r}\right)^{*}\left(\mathscr{M}_{p}\right) \cdot\left\{\left(e_{1} ; \ldots ; e_{1}\right), \ldots,\left(e_{p} ; \ldots ; e_{p}\right)\right\}
\end{aligned}
$$

Given $u \in \mathscr{M}_{p},\left(f_{1} ; \ldots ; f_{r}\right)^{*}(u)$ is defined to be $\left(f_{1}^{*}(u) ; \ldots ; f_{r}^{*}(u)\right)$, and we therefore have explicitly:

$$
\left(f_{1} ; \ldots ; f_{r}\right)^{*}(u) \cdot\left(e_{i} ; \ldots ; e_{i}\right)=\left(f_{1}^{*}(u) e_{i} ; \ldots ; f_{r}^{*}(u) e_{i}\right) .
$$

Note the restricted form of the left tangent space in comparison with the right tangent space which is the direct sum of each of the individual right tangent spaces $L \mathscr{R} \cdot f_{i}$. This is related to point (i) of the preceding remark.

\subsection{Classification Techniques}

We will use the standard philosophy of reducing all classifications to calculations in jet-spaces. The problem is then one of Lie groups acting on affine spaces and techniques such as Mather's Lemma [11, Lemma 3.1], and Complete Transversals [2], may be applied. The technical details are merely a generalisation of those from the familiar monogerm case. We will just state the results which provide the machinery behind the classification. From the discussion above and the analogy with the standard monogerm case, the notation used should be clear. It will suffice to use one of the more elementary of the complete transversal theorems presented in [2], namely the use of $\mathscr{A}$ equivalences whose 1 -jet is the identity.

The standard $k$-jet-space $\mathscr{M}_{n} \mathscr{E}(n, p) / \mathscr{M}_{n}^{k+1} \mathscr{E}(n, p)$ is denoted $J^{k}(n, p)$. Since we choose to represent the space of all $r$-multigerms as $\oplus_{1}^{r} \mathscr{M}_{n} \mathscr{E}(n, p)$, the corresponding multijet space can then be realised as $\oplus_{1}^{r} J^{k}(n, p)$. Let $\mathscr{R}_{k}$ be the normal subgroup of $\mathscr{R}$ consisting of those germs whose $k$-jet is equal to that of the identity; and similarly for $\mathscr{L}$. We consider the (multi) $k$-jet-groups $\left(\oplus_{1}^{r} \mathscr{R} / \mathscr{R}_{k}\right) \oplus\left(\mathscr{L} / \mathscr{L}_{k}\right)$ and $\left(\oplus_{1}^{r} \mathscr{R}_{1} / \mathscr{R}_{k}\right) \oplus\left(\mathscr{L}_{1} / \mathscr{L}_{k}\right)$ denoted ${ }_{r} J^{k} \mathscr{A}$ and ${ }_{r} J^{k} \mathscr{A}_{1}$ respectively (or simply $J^{k} \mathscr{A}$ and $J^{k} \mathscr{A}_{1}$ for short). These are Lie groups and act on the affine space $\oplus_{1}^{r} J^{k}(n, p)$; see [10, Section 7]. Let $H^{k}$ denote the image of $\oplus_{1}^{r} \mathscr{M}_{n}^{k} \mathscr{E}(n, p)$ in $\oplus_{1}^{r} J^{k}(n, p)$, the vector subspace of $\oplus_{1}^{r} J^{k}(n, p)$ consisting of the homogeneous jets of degree $k$.

TheOREM 2.1. Let $f$ be a multigerm $\mathrm{R}^{n}, S \rightarrow \mathrm{R}^{p}, 0$. If $T \subset H^{k+1}$ is a vector subspace of $H^{k+1}$ such that

$$
H^{k+1} \subset T+L\left({ }_{r} J^{k+1} \mathscr{A}_{1}\right) \cdot j^{k} f
$$


then every $(k+1)$-jet $F$ with $j^{k} F=j^{k} f$ is in the same ${ }_{r} J^{k+1} \mathscr{A}_{1}$-orbit as some $(k+1)$-jet of the form $j^{k} f+t$, for some $t \in T$.

For determinacy and complete transversal calculations we must work with the tangent space $L \mathscr{A}_{1}$, or more precisely the Lie algebra $L\left({ }_{r} J^{k} \mathscr{A}_{1}\right)$. This is just the projection of $L \mathscr{A}_{1}$ into the jet-space and, in coordinate form, $L \mathscr{A}_{1}$ is given by the expressions (1) above, only now the maximal ideals $\mathscr{M}_{n}$ and $\mathscr{M}_{p}$ are raised to the power of 2 .

Theorem 2.1 allows us to classify jets with respect to ${ }_{r} J^{k} \mathscr{A}_{1}$-equivalence (and therefore ${ }_{r} J^{k} \mathscr{A}$-equivalence) inductively at the jet level. This provides an $\mathscr{A}$-classification of finitely determined multigerms. From [10], a multigerm $f$ is finitely determined if and only if it has finite $\mathscr{A}$-codimension (or equivalently finite $\mathscr{A}_{e}$-codimension). Applying the methods of [3] (or indeed Theorem 2.1 above) we obtain the following determinacy criterion.

\section{THEOREM 2.2. A multigerm $f$ of multiplicity $r$ is $k$-determined if}

$$
\oplus_{1}^{r} \mathscr{M}_{n}^{k+1} \mathscr{E}(n, p) \subset L \mathscr{A}_{1} \cdot f .
$$

To make this suitable for implementation on a computer we need to reduce it to a question in some appropriate jet-space.

Corollary 2.3. A multigerm $f$ of multiplicity $r$ is $k$-determined if $\oplus_{1}^{r} \mathscr{M}_{n}^{k+1} \mathscr{E}(n, p) \subset L \mathscr{A}_{1} \cdot f+f^{*}\left(\mathscr{M}_{p}\right)\left(\oplus_{1}^{r} \mathscr{M}_{n}^{k+1} \mathscr{E}(n, p)\right)+\oplus_{1}^{r} \mathscr{M}_{n}^{2 k+2} \mathscr{E}(n, p)$.

Remark 2. Corollary 2.3 follows from [3, Lemma 2.6], extended to the multigerm case. The proof is similar to the original, only now we work with the finitely generated $\oplus_{1}^{r} \mathscr{E}_{n}$-module $\oplus_{1}^{r} \mathscr{E}(n, p)$ and the $\mathscr{E}_{p}$-module structure is induced via the ring homomorphism $\left(f_{1} ; \ldots ; f_{r}\right)^{*}: \mathscr{E}_{p} \rightarrow \oplus_{1}^{r} \mathscr{E}_{n}$. The technical tools needed in this (and many of the other standard results, such as the determinacy and unfolding theorems) are Nakayama's lemma and The Preparation Theorem and both hold in this setting. Note that the foundational work of Mather applied to the case of multigerms; for example, see [10] for the determinacy theorems and The Preparation Theorem.

\subsection{Codimension Restrictions}

We now return to the specific case of multigerms $\mathrm{R}^{2}, S \rightarrow \mathrm{R}^{3}, 0$. From [6] the condition for a stratum of the jet space ${ }_{r} J^{k}(2,3)$ to be relevant to the kinematics problem is that it must have $\mathscr{A}$-codimension $\leq 6-r$, where $r$ is the number of branches (multiplicity) of the multigerm. This tells us immediately that we need look for multigerms with at most 6 branches. In Section 3 we classify 
$r$-germs with these conditions in mind, stopping when we have found all of the relevant strata. The following proposition helps rule out many of the initial branches of the classification tree on codimension grounds.

Proposition 2.4. Let $f$ be the multigerm $\left(g_{1} ; \ldots ; g_{r} ; h_{1} ; \ldots ; h_{s}\right)$ of multiplicity $r+s$. Consider the associated multigerms $g=\left(g_{1} ; \ldots ; g_{r}\right)$ and $h=\left(h_{1} ; \ldots ; h_{s}\right)$ of multiplicity $r$ and s respectively. Then

$$
\mathscr{A}-\operatorname{codim}(f) \geq \mathscr{A}-\operatorname{codim}(g)+\mathscr{A}-\operatorname{codim}(h) .
$$

Proof. From the definition of the $\mathscr{A}$ tangent space given in equations (1) we see that there is a natural inclusion $L \mathscr{A} \cdot f \rightarrow L \mathscr{A} \cdot g \oplus L \mathscr{A} \cdot h$. Thus, there is a natural surjection

$$
\begin{aligned}
\oplus_{1}^{r+s} \mathscr{M}_{n} \mathscr{E}(n, p) / L \mathscr{A} \cdot f \rightarrow \oplus_{1}^{r+s} \mathscr{M}_{n} \mathscr{E}(n, p) /(L \mathscr{A} \cdot g \oplus L \mathscr{A} \cdot h) \\
\cong\left(\oplus_{1}^{r} \mathscr{M}_{n} \mathscr{E}(n, p) / L \mathscr{A} \cdot g\right) \oplus\left(\oplus_{1}^{s} \mathscr{M}_{n} \mathscr{E}(n, p) / L \mathscr{A} \cdot h\right) .
\end{aligned}
$$

Looking at the real dimension of these spaces we obtain the result.

Corollary 2.5. Let $f$ be the multigerm $\left(f_{1} ; \ldots ; f_{r}\right)$ then

$$
\mathscr{A}-\operatorname{codim}(f) \geq \sum_{i=1}^{r} \mathscr{A}-\operatorname{codim}\left(f_{i}\right) .
$$

(The codimension referred to on the right hand side of the above inequality is, of course, the standard $\mathscr{A}$-codimension of a monogerm $f_{i}$.)

We make the following preliminary observations. For monogerms $\mathrm{R}^{2}, 0 \rightarrow$ $\mathrm{R}^{3}, 0$ the possible 1 -jets are $(x, y, 0),(x, 0,0)$ and $(0,0,0)$ of $J^{1} \mathscr{A}$-codimension 0,2 and 6 , respectively. Therefore none of the multigerms we are interested in can have a branch with zero 1-jet. In the cases $r=3$ and $r=4$ at most one branch cannot be immersive. In the cases $r=5$ and $r=6$ all branches must be immersive. Further restrictions on the classification of $r$-multigerms transpire once the classification of $(r-1)$-multigerms has been achieved.

Since the $J^{k} \mathscr{A}$-codimension is increasing with $k$ we achieve the bounds on codimension using inductive classification at the jet-level. More precisely, given a multigerm $f$, the natural projection $\pi:\left(J^{k+1} \mathscr{A}\right) \cdot j^{k+1} f \rightarrow\left(J^{k} \mathscr{A}\right)$. $j^{k} f$ from the $J^{k+1} \mathscr{A}$ orbit of $f$ to the $J^{k} \mathscr{A}$ orbit of $f$, is surjective. Therefore $\pi^{-1}\left(\left(J^{k} \mathscr{A}\right) \cdot j^{k} f\right)$ is a submanifold of $J^{k+1}(n, p)$ with codimension equal to the codimension of $\left(J^{k} \mathscr{A}\right) \cdot j^{k} f$ in $J^{k}(n, p)$. But $\pi^{-1}\left(\left(J^{k} \mathscr{A}\right)\right.$. $\left.j^{k} f\right) \supset\left(J^{k+1} \mathscr{A}\right) \cdot j^{k+1} f$ and we have the following well known result: $J^{k} \mathscr{A}$-codim $(f) \leq J^{k+1} \mathscr{A}$-codim $(f)$. Finally, if $f$ is $k$ - $\mathscr{A}$-determined then, by the determinacy theorems of [3], there exists some unipotent $\operatorname{subgroup} \mathscr{G}$ of 
$\mathscr{A}$ such that $\oplus_{1}^{r} \mathscr{M}_{n}^{k+1} \mathscr{E}(n, p) \subset L \mathscr{G} \cdot f$, and in particular $\oplus_{1}^{r} \mathscr{M}_{n}^{k+1} \mathscr{E}(n, p) \subset$ $L \mathscr{A} \cdot f$. It then follows that $J^{k} \mathscr{A}-\operatorname{codim}(f)=\mathscr{A}$-codim $(f)$.

Thus, it is natural to work with $\mathscr{A}$-codimension at the jet-level. In Section 4 we study the geometry of our multigerms via versal unfoldings. The following relation between $\mathscr{A}$ - and $\mathscr{A}_{e}$-codimension is useful.

Proposition 2.6. Let $f: \mathrm{R}^{n}, S \rightarrow \mathrm{R}^{p}, 0$ be an $\mathscr{A}$-finite multigerm of multiplicity $r$. If $f$ is not stable then the following relation holds: $\mathscr{A}_{e}$-codim $=$ $\mathscr{A}$-codim $+r(p-n)-p$.

A proof appears in the unpublished notes of Wilson [16] and, in the case $r=1$, in the survey article of Wall [15, p.510]. We remark that our codimension restrictions $\mathscr{A}$-codim $\leq 6-r$ are therefore equivalent to the single condition $\mathscr{A}_{e}$-codim $\leq 3$ for all multigerms under consideration.

\section{Classification}

In this section we describe the classification of multigerms up to the codimension restrictions discussed in Section 2.2. The results are summarised in Theorem 1.2. By taking the classification further we obtain series which provide us with the full list of simple multigerms given in Theorem 1.1. To give full details of the calculations would extend the paper, whose main concern is obtaining a stratification of the jet-space with respect to codimension, by a significant length. We provide a summary of the classification process which should act as a suitable guide for anyone keen enough to repeat any parts of the calculation! However, for brevity, the full details are omitted.

We name the multigerms by exploiting existing notation in a systematic way. The notation of Mond is used to describe each branch of the multigerm ( $A_{0}$ will represent an immersed branch). In addition, if the branches intersect in a non-transverse manner then the contact type of their intersection is appended (following the symbol $\mid$ ). For example, the bigerm consisting of 2 transverse sheets is denoted $A_{0} A_{0}$, whereas the case of 2 sheets intersecting with $A_{k}^{ \pm}$contact is denoted $A_{0} A_{0} \mid A_{k}^{ \pm}$(shortened to $A_{0}^{2}$ and $A_{0}^{2} \mid A_{k}^{ \pm}$, respectively). For trigerms the contact type applies to all three branches of the trigerm (in all of our examples this is the contact of the third branch with the intersection curve of the first two) unless brackets dictate otherwise. Thus, $A_{0}^{3}$ represents the transverse triple point and $A_{0}^{3} \mid A_{k}$ denotes the case where two sheets intersect transversely and the third makes $(k+1)$-order contact with their intersection curve (specifically, the contact map is $A_{k},\left(y, x^{k+1}\right)$ in this case). When two sheets intersect with $A_{k}^{ \pm}$-contact (as a bigerm) and the third sheet is transverse to both we use the notation $\left(A_{0}^{2} \mid A_{k}^{ \pm}\right)\left(A_{0}\right) \mid A_{1}$. The final $A_{1}$ refers to the contact type of all three branches, but since this is the least degenerate case 
(contact to order $A_{1}$ or higher is inevitable) we drop this $A_{1}$ to simplify the notation. The full notation is required to distinguish higher contact types such as $\left(A_{0}^{2} \mid A_{2}\right)\left(A_{0}\right) \mid A_{2}$. Finally, in a few cases the branch and contact type are enough to describe only a stem. A subscript is therefore appended to the notation describing the whole series. The transverse intersection of a sheet and a cross-cap $\left(A_{0} S_{0}\right)_{k}$ and $A_{\infty}$ intersection $\left(A_{0} S_{0} \mid A_{\infty}\right)_{k}$ are examples of this kind.

The rest of this section will summarise the details of the classification. We will describe the stratification of the low degree jet-spaces but, for brevity, describe how the classification proceeds only along a few selected branches. We will adhere to the following conventions. Coordinates in the source will be denoted $(x, y)$ (extending this to $(X, Y),(\bar{x}, \bar{y})$, etc., to help distinguish each branch); while coordinates in the target will be denoted $(u, v, w)$. We will use the term complete transversal to mean the affine space $f+T$ (and occasionally just give a basis for $T$ ) referred to in Theorem 2.1. At each given jet-level $J^{k}$ we will summarise all the relevant jets in the form of a table, listing the jets in order of increasing $J^{k} \mathscr{A}$-codimension. The columns of the table contain: a numeric label for future reference to the jet; the jet itself; the $J^{k} \mathscr{A}$-codimension of the jet; and finally comments - for example, the symbol \# will be used to indicate the jet is ruled out of further consideration on codimension grounds.

As mentioned above, for brevity we present a summary of the classification and, as such, only provide a guide to the reader on how to produce the list of singularities in Table 1. In particular, in most cases it is a straightforward matter to identify adjacencies which prove that a jet is non-simple. For example, in Section 3.1 (bigerms), Case 3.2.4 deforms to 3.2.3, all of whose germs are found to be modular. Such considerations are implicit at several stages of the classification of the simple multigerms. However, for completeness we mention the slightly more involved Case 4 (from Section 3.1 also). It is not entirely clear from the details describing this case that all of the simples are captured. In particular, the 2-jet $\left(x, y, 0 ; X, Y^{2}, X^{2}\right)$, of $\mathscr{A}$-codimension 5 , must be considered further. It turns out that the jets lying over this case are modular families and the following series.

$$
\begin{aligned}
& A_{0} S_{k}^{ \pm} \mid A_{2} \quad\left(x, y, 0 ; X, Y^{2}, X^{2}+Y^{3} \pm X^{k+1} Y\right) \\
& k \geq 1, \quad(k+2) \text {-determined, } \quad \mathscr{A} \text {-codim }=k+4 .
\end{aligned}
$$

Adjacencies with $A_{0} S_{1} \mid A_{1}^{ \pm}$confirm that all members of this series are nonsimple, thus completing the treatment of this case. Finally, we remark that to prove the singularities in Table 1 are simple one only has to establish a set of all possible adjacencies, and not the more difficult task of identifying which adjacencies actually exist. 


\subsection{Classification of Bigerms}

Here we classify bigerms with $\mathscr{A}$-codimension $\leq 4$. Recall that no such bigerm can have a branch with zero 1 -jet. Up to $\mathscr{A}$-equivalence the possible 1 -jets are as follows.

$\begin{array}{lccc} & 1 \text {-jet } & J^{1} \mathscr{A} \text {-codim } & \\ \mathbf{1} & (x, y, 0 ; 0, X, Y) & 0 & 1 \text {-det } \\ \mathbf{2} & (x, y, 0 ; X, Y, 0) & 2 & \\ \mathbf{3} & (x, y, 0 ; 0,0, X) & 2 & \\ \mathbf{4} & (x, y, 0 ; X, 0,0) & 3 & \\ \mathbf{5} & (x, 0,0 ; 0, X, 0) & 4 & \\ \mathbf{6} & (x, 0,0 ; X, 0,0) & 6 & \#\end{array}$

Case 2. The bigerm can be written in the form $(x, y, 0 ; X, Y, \phi(X, Y))$. For such bigerms we refer to $\phi$ as the separation function and have the following result.

THEOREM 3.1. [12, Thm. 7.2], Bigerms of immersions are classified for $\mathscr{A}$ by the $\mathscr{K}$-classes of the separation function $\phi(X, Y)$. The $\mathscr{A}_{e}$-codimension (which equals the $\mathscr{A}$-codimension -1) of the bigerm is given by the $\mathscr{K}_{e}$-codimension of $\phi$.

Using this we find that the bigerms of immersions with $\mathscr{A}$-codimension $\leq 4$ are $A_{0}^{2}\left|A_{1}^{ \pm}, A_{0}^{2}\right| A_{2}$ and $A_{0}^{2} \mid A_{3}^{ \pm}$. So the first part of the stratification of $\oplus_{1}^{2} J^{k}(2,3)$ is given by the first four entries in Table 2. We also obtain the simple series $A_{0}^{2}\left|A_{k}^{ \pm}, A_{0}^{2}\right| D_{k}^{ \pm}, A_{0}^{2}\left|E_{6}, A_{0}^{2}\right| E_{7}$ and $A_{0}^{2} \mid E_{8}$ in Table 1 . The contact type of the two branches is just the $\mathscr{K}$-class of the separation function and appears in the notation.

Case 3. A 2-transversal is $\left(x, y, 0 ; a Y^{2}+b X Y, c Y^{2}+d X Y, X\right)$. Here we will describe the diffeomorphic changes of coordinates in the source and target which reduce the above family to four possible non- $\mathscr{A}$-equivalent 2 -jets. Calculating the $J^{2} \mathscr{A}$-codimension for each verifies that these represent four distinct orbits.

First suppose $a \neq 0$. We can scale $a$ to equal 1 and then remove the $c Y^{2}$ term via the coordinate change $v \mapsto v-c u$. This leaves us with $j^{2} f=$ $\left(x, y-c x, 0 ; Y^{2}+b X Y,(d-c b) X Y, X\right)$, but using a change of coordinates $y \mapsto y+c x$ in the source of the first branch gives $j^{2} f=\left(x, y, 0 ; Y^{2}+\right.$ $b X Y,(d-c b) X Y, X)$. Now we can write $Y^{2}+b X Y$ as $(Y+b X / 2)^{2}-b^{2} X^{2} / 4$, and change coordinates in the source of the second branch via $Y \mapsto Y-b X / 2$ to get $j^{2} f=\left(x, y, 0 ; Y^{2}-b^{2} X^{2} / 4,(d-b c) X Y-(d-b c) b X^{2} / 2, X\right)$. (This is known as completing the square, a special case of the Tschirnhaus transformation.) But we can change coordinates in the target via $u \mapsto u+$ 
$b^{2} w^{2} / 4$ and $v \mapsto v+(d-b c) b w^{2} / 2$ (which will not affect the first branch) to get $j^{2} f=\left(x, y, 0 ; Y^{2},(d-b c) X Y, X\right)$. Then either $d-b c$ is zero, or it can be scaled to equal 1 .

By symmetry, a similar argument holds if $c \neq 0$. Finally, consider $a=c=$ 0 . If $b \neq 0$ we can scale it to be 1 and then use the target change of coordinates $v \mapsto v-d u$ followed by a change of coordinates in the source of the first branch to get $j^{2} f=(x, y, 0 ; X Y, 0, X)$. If $b=0$ but $d \neq 0$ we arrive at the same 2-jet, leaving the final possibility: $a=b=c=d=0$.

In summary, the possible 2-jets are as follows.

\begin{tabular}{llc} 
& \multicolumn{1}{c}{ 2-jet } & $J^{2} \mathscr{A}$-codim \\
$\mathbf{3 . 1}$ & $\left(x, y, 0 ; Y^{2}, X Y, X\right)$ & 2 \\
$\mathbf{3 . 2}$ & $\left(x, y, 0 ; 0, Y^{2}, X\right)$ & 3 \\
$\mathbf{3 . 3}$ & $(x, y, 0 ; 0, X Y, X)$ & 4 \\
$\mathbf{3 . 4}$ & $(x, y, 0 ; 0,0, X)$ & 6
\end{tabular}

Case 3.1. For this case we appeal to the following theorem. This gives the series $\left(A_{0} S_{0}\right)_{k}$ and corresponding entries in Table 2 .

Theorem 3.2. [12, Thm. 7.5] (i) The bigerm ( $\left.x, y, 0 ; Y^{2}, X Y+Y^{2 k+1}, X\right)$ is $(2 k+1)$-determined and of $\mathscr{A}_{e}$-codimension $k$, where $k \geq 1$.

(ii) Any finitely determined bigerm consisting of an immersion and a crosscap, meeting transversely, is equivalent to one of the germs defined in (i).

Case 3.2. A 3-transversal is $\left(x, y, 0 ; a Y^{3}+b X^{2} Y, Y^{2}, X\right)$ and after further simplification similar to that above we obtain the following 3 -jets.

\begin{tabular}{llcc} 
& \multicolumn{1}{c}{3 -jet } & $J^{3} \mathscr{A}$-codim & \\
3.2.1 & $\left(x, y, 0 ; Y^{3} \pm X^{2} Y, Y^{2}, X\right)$ & 3 & 3 -det \\
3.2.2 & $\left(x, y, 0 ; Y^{3}, Y^{2}, X\right)$ & 4 & \\
3.2.3 & $\left(x, y, 0 ; X^{2} Y, Y^{2}, X\right)$ & 4 & \\
3.2.4 & $\left(x, y, 0 ; 0, Y^{2}, X\right)$ & 5 & $\#$
\end{tabular}

Case 3.2.2. Further calculations give the series $A_{0} S_{k}^{ \pm}$.

Case 3.2.3. First note that the critical sets of the second branch of 3.2 .2 and 3.2.3 are not diffeomorphic, so that these multigerms are not $\mathscr{A}$-equivalent. A 4-transversal is $\left(x, y, 0 ; X^{2} Y+a X Y^{3}, Y^{2}, X\right)$ and we may reduce this to the following two cases.

$$
\begin{array}{ll}
\multicolumn{1}{c}{\text { 4-jet }} & J^{4} \mathscr{A} \text {-codim } \\
\left(x, y, 0 ; X^{2} Y+X Y^{3}, Y^{2}, X\right) & 4 \\
\left(x, y, 0 ; X^{2} Y, Y^{2}, X\right) & 5
\end{array}
$$


Continuing with the first, a 5-transversal is $\left(x, y, 0 ; X^{2} Y+X Y^{3}+a Y^{5}, Y^{2}, X\right)$; denote this family $f_{a}$. We find that the $J^{5} \mathscr{A}$-codimension is 5 for all values of $a$, and that $\left(0,0,0 ; 0, Y^{5}, 0\right) \notin L\left(J^{5} \mathscr{A}\right) \cdot f_{a}$. So $a$ is a genuine modulus and the codimension of the stratum is 4 . Further, $f_{a}$ is 5-determined for $a \neq 0, \frac{1}{4}$ and this provides the first unimodular family in our classification. The exceptional cases are of codimension at least 5 and can be discarded.

Case 3.3. Returning now to the 2-jets, a 3-transversal for $(x, y, 0 ; 0, X Y, X)$ is $f_{a, b, c}=\left(x, y, 0 ; a Y^{3}+b X Y^{2}, X Y+c Y^{3}, X\right)$. Direct computation of $L\left(J^{3} \mathscr{A}\right) \cdot f_{a, b, c}$ shows that if $a=0$ then the $J^{3} \mathscr{A}$-codimension is at least 5. We can therefore ignore this case and assume that (after scaling) $a=1$. We then apply Mather's Lemma and see that the family $f_{b, c}=\left(x, y, 0 ; Y^{3}+b X Y^{2}, X Y+\right.$ $\left.c Y^{3}, X\right)$ is trivial in $b$ and $c$. Specifically, we show that the $J^{3} \mathscr{A}$-codimension of $f_{b, c}$ is 4 and that the vectors tangent to this family, $\left(0,0,0 ; X Y^{2}, 0,0\right)$ and $\left(0,0,0 ; 0, Y^{3}, 0\right)$, are contained in $L\left(J^{3} \mathscr{A}\right) \cdot f_{b, c}$ for all values of $b$ and $c$. Thus we have one $J^{3} \mathscr{A}$-orbit to consider, namely $\left(x, y, 0 ; Y^{3}, X Y, X\right)$.

Continuing, a 4-transversal is $\left(x, y, 0 ; Y^{3}+a Y^{4}, X Y+b Y^{4}, X\right)$ and this family has $J^{4} \mathscr{A}$-codimension $\leq 4$ only if $a \neq 0$ and $b \neq 0$. We can therefore assume that (after scaling) $a$ and $b$ are equal to 1 , giving $\left(x, y, 0 ; Y^{3}+Y^{4}, X Y+\right.$ $\left.Y^{4}, X\right)$.

A 5-transversal is $\left(x, y, 0 ; Y^{3}+Y^{4}, X Y+Y^{4}+a Y^{5}, X\right)$. Here $a$ is a modulus and each member of the family is 5-determined and of $J^{5} \mathscr{A}$-codimension 5 provided $a \neq 1$. The codimension of the whole stratum is 4 .

Case 4. The classification of branches over this 1-jet follows similar arguments to those for Case 3; we will just add the following observations. A 2-transversal is $\left(x, y, 0 ; X, a Y^{2}+b X Y, c Y^{2}+d X Y+e X^{2}\right)$. After further simplification we obtain four 2-jets of $\mathscr{A}$-codimension $\leq 4$ which, in turn, give rise to the types $A_{0} S_{0} \mid A_{1}^{ \pm}$through to $\left(A_{0} S_{0} \mid A_{\infty}\right)_{2}$ and the series $A_{0} S_{0} \mid A_{k}^{ \pm}$and $\left(A_{0} S_{0} \mid A_{\infty}\right)_{k}$ in Tables 2 and 1. (See the remarks at the end of the introduction to Section 3 also.)

Case 5. A 2-transversal is $\left(x, a y^{2}+b x y, c y^{2}+d x y ; e Y^{2}+f X Y, X, g Y^{2}+\right.$ $h X Y)$. We find there is only one orbit of $J^{2} \mathscr{A}$-codimension $\leq 4$, namely $\left(x, x y, y^{2} ; X Y, X, Y^{2}\right)$. On continuing the classification, the only relevant case to arise from this jet is type $S_{0} S_{0}$. This completes the classification of the bigerms.

\subsection{Classification of Trigerms}

We know that a trigerm of $\mathscr{A}$-codimension $\leq 3$ must have at least two immersed branches and that no branch can have zero 1-jet. Certainly we can have three transverse immersions: this is Case 1 below. Now let us suppose that the 
first two branches are immersions. The possibilities are $(x, y, 0 ; X, 0, Y)$ and $(x, y, 0 ; X, Y, 0)$. Direct computation shows there are nine trigerms having two immersed branches, those of $\mathscr{A}$-codimension $\leq 3$ are as follows.

$\begin{array}{cccc} & & J^{1} \mathscr{A} \text {-codim } & \\ \mathbf{1} & (x, y, 0 ; X, 0, Y ; 0, \bar{x}, \bar{y}) & 0 & 1 \text {-det } \\ \mathbf{2} & (x, y, 0 ; X, 0, Y ; \bar{x}, \bar{y}, \bar{y}) & 1 & \\ \mathbf{3} & (x, y, 0 ; X, 0, Y ; \bar{x}, \bar{y}, 0) & 2 & \\ \mathbf{4} & (x, y, 0 ; X, 0, Y ; 0, \bar{x}, \bar{x}) & 2 & \\ \mathbf{5} & (x, y, 0 ; X, 0, Y ; 0, \bar{x}, 0) & 3 & \end{array}$

Case 2. Further calculations give the series $A_{0}^{3} \mid A_{k}$.

Case 3. A 2-transversal is $\left(x, y, 0 ; X, 0, Y ; \bar{x}, \bar{y}, a \bar{y}^{2}+b \bar{x} \bar{y}+c \bar{x}^{2}\right)$. We will show how to apply elementary coordinate changes to reduce this family to five non- $J^{3} \mathscr{A}$-equivalent 3 -jets.

First suppose $c \neq 0$, scale it to equal 1 and then complete the square in the last component to give $\left(x, y, 0 ; X, 0, Y ; \bar{x}+\alpha \bar{y}, \bar{y}, \bar{x}^{2}+\beta \bar{y}^{2}\right)$ for some $\alpha$ and $\beta$. Now change coordinates via $u \mapsto u-\alpha v$ to get $(x-\alpha y, y, 0 ; X, 0, Y ; \bar{x}, \bar{y}$, $\left.\bar{x}^{2}+\beta \bar{y}^{2}\right)$. Then a change in the source coordinates $(x, y)$ and $(\bar{x}, \bar{y})$ gives the 2-jet $\left(x, y, 0 ; X, 0, Y ; \bar{x}, \bar{y}, \bar{x}^{2} \pm \bar{y}^{2}\right)$ or $\left(x, y, 0 ; X, 0, Y ; \bar{x}, \bar{y}, \bar{x}^{2}\right)$.

If $c=0$ but $b \neq 0$ then we can write the 2-jet as $(x, y, 0 ; X, 0, Y ; \bar{x}, \bar{y}, \bar{y}(\bar{x}+$ $\alpha \bar{y})$ ). Changing the source coordinates $(\bar{x}, \bar{y})$ gives $(x, y, 0 ; X, 0, Y ; \bar{x}-\alpha \bar{y}, \bar{y}$, $\bar{y} \bar{x})$. As before we can then reduce to give $(x, y, 0 ; X, 0, Y ; \bar{x}, \bar{y}, \bar{x} \bar{y})$.

Finally, $c=b=0$ gives the remaining two cases. The complete stratification is as follows.

$$
\text { 2-jet } \quad J^{2} \mathscr{A} \text {-codim }
$$

$\begin{array}{llcc}\text { 3.1 } & \left(x, y, 0 ; X, 0, Y ; \bar{x}, \bar{y}, \bar{x}^{2} \pm \bar{y}^{2}\right) & 2 & 2-\operatorname{det} \\ \text { 3.2 } & \left(x, y, 0 ; X, 0, Y ; \bar{x}, \bar{y}, \bar{x}^{2}\right) & 3 & \\ \text { 3.3 } & (x, y, 0 ; X, 0, Y ; \bar{x}, \bar{y}, \bar{x} \bar{y}) & 3 & \\ \text { 3.4 } & \left(x, y, 0 ; X, 0, Y ; \bar{x}, \bar{y}, \bar{y}^{2}\right) & 4 & \# \\ \text { 3.5 } & (x, y, 0 ; X, 0, Y ; \bar{x}, \bar{y}, 0) & 5 & \#\end{array}$

Case 3.2. Further calculations give the series $\left(A_{0}^{2} \mid A_{k}^{ \pm}\right)\left(A_{0}\right)$.

Case 3.3. Further calculations give the series $\left(A_{0}^{2} \mid A_{\infty}\right)\left(A_{0}\right) \mid A_{k}$. (Note that we specify $k \geq 2$ in this series because for $k=1$ the resulting multigerm is $\mathscr{A}$-equivalent to Case 3.1.)

Case 3.4. Although this case is ruled out on codimension grounds, we must consider it further to complete the list of simple multigerms. A 3-transversal is $\left(x, y, 0 ; X, 0, Y ; \bar{x}, \bar{y}, \bar{y}^{2}+a \bar{x}^{2} \bar{y}+b \bar{x}^{3}\right)$. If $b \neq 0$ then, applying Mather's 
Lemma, we can reduce it to equal 1 and also find that the family in $a$ is trivial. The resulting jet $\left(A_{0}^{2} \mid A_{2}\right)\left(A_{0}\right) \mid A_{2}$ is 3 -determined of $\mathscr{A}$-codimension 4 . The case $b=0$ gives rise to non-simples and is not considered further.

Case 4. A 2-transversal is ( $\left.x, y, 0 ; X, 0, Y ; a \bar{y}^{2}+b \bar{x} \bar{y}, \bar{x}, \bar{x}+c \bar{y}^{2}+d \bar{x} \bar{y}\right)$. Using arguments similar to those of previous examples we find that there are three 2 -jets of $\mathscr{A}$-codimension $\leq 3$ which, in turn, give rise to the types $A_{0}^{2} S_{0} \mid A_{1}$ through to $A_{0}^{2} S_{0} \mid A_{2}$ in Table 2 .

Case 5. A 2-transversal is ( $\left.x, y, 0 ; X, 0, Y ; a \bar{y}^{2}+b \bar{x} \bar{y}, \bar{x}, c \bar{y}^{2}+d \bar{x} \bar{y}+e \bar{x}^{2}\right)$. The only orbit with $J^{2} \mathscr{A}$-codimension $\leq 3$ is found to be $(x, y, 0 ; X, 0, Y ; \bar{x} \bar{y}$, $\bar{x}, \bar{y}^{2} \pm \bar{x}^{2}$ ). Further calculations give the following series. (We remark that the members of this series are non-simple.) The case $k=1$ provides the final trigerm in Table 2 and our classification of trigerms is complete.

$$
\begin{aligned}
\left(A_{0} S_{0} \mid A_{1}^{ \pm}\right)\left(A_{0}\right)_{k} \quad\left(x, y, 0 ; X, 0, Y ; \bar{x} \bar{y}+\bar{y}^{2 k+1}, \bar{x}, \bar{y}^{2} \pm \bar{x}^{2}\right) \\
\quad k \geq 1, \quad(2 k+1) \text {-determined, } \quad \mathscr{A} \text {-codim }=k+2 .
\end{aligned}
$$

\subsection{Classification of Four-germs}

We need to consider 4-germs with $\mathscr{A}$-codimension $\leq 2$. We know that any 4germ $\left(f_{1} ; f_{2} ; f_{3} ; f_{4}\right)$ relevant to our problem must have at least three immersed branches and can assume that these are $f_{1}, f_{2}$ and $f_{3}$; in addition, $f_{4}$ cannot have zero 1 -jet. If $f_{4}$ is immersed then $\left(f_{1} ; f_{2} ; f_{3}\right)$ can be any of the trigerms (1), (2) or (3) in Section 3.2. However, direct computation shows there are two 4-germs of type (2), each of $\mathscr{A}$-codimension 3 , and two 4-germs of type (3), each of $\mathscr{A}$-codimension 4 ; the remaining germs being equivalent to those obtained from type (1). Therefore the only relevant case is type (1) and we

\begin{tabular}{|c|c|c|}
\hline & 1-jet & $J^{1} \mathscr{A}$-codim \\
\hline 1 & $(x, y, 0 ; X, 0, Y ; 0, \bar{x}, \bar{y} ; \bar{X}, \bar{Y}, \bar{X}+\bar{Y})$ & 0 \\
\hline & $(x, y, 0 ; X, 0, Y ; 0, \bar{x}, \bar{y} ; \bar{X}, \bar{Y}, \bar{Y})$ & 1 \\
\hline & $(x, y, 0 ; X, 0, Y ; 0, \bar{x}, \bar{y} ; \bar{X}, \bar{Y}, 0)$ & 2 \\
\hline
\end{tabular}
obtain the following.

Finally, if $f_{4}$ is $\mathscr{A}$-equivalent to $(x, 0,0)$ then, from Proposition $2.4,\left(f_{1} ; f_{2} ; f_{3}\right)$ must be $\mathscr{A}$-equivalent to the trigerm (1). However, the only 4-germ of this type is found to be $(x, y, 0 ; X, 0, Y ; 0, \bar{x}, \bar{y} ; \bar{X}, 0,0)$, having $\mathscr{A}$-codimension 4 .

Case 2. Further calculations give the series $\left(A_{0}^{3} \mid A_{k}\right)\left(A_{0}\right)$.

Case 3. A 2-transversal is $\left(x, y, 0 ; X, 0, Y ; 0, \bar{x}, \bar{y} ; \bar{X}, \bar{Y}, a \bar{Y}^{2}+b \bar{X} \bar{Y}+\right.$ $c \bar{X}^{2}$ ). Using elementary coordinate changes and Mather's lemma we obtain 
only one 2-jet relevant to our problem, namely $(x, y, 0 ; X, 0, Y ; 0, \bar{x}, \bar{y} ; \bar{X}, \bar{Y}$, $\left.\bar{Y}^{2}+\bar{X} \bar{Y}+c \bar{X}^{2}\right)$

The coefficient $c$ is a genuine modulus and the whole stratum has codimension 2. If $c \neq 0, \frac{1}{4}$ the 4-germ is 2-determined. (The isolated cases $c=0$ and $c=\frac{1}{4}$ are ruled out, being of too high a codimension.)

\subsection{Classification of Five-germs}

We need to consider 5-germs with $\mathscr{A}$-codimension $\leq 1$. From Proposition 2.4, all branches must be immersed and the 4-germ formed by the first four branches (say) must be of type (1) or (2) in Section 3.3. We find that there are five cases, with two having $\mathscr{A}$-codimension $\leq 1$.

1 -jet

$1(x, y, 0 ; X, 0, Y ; 0, \bar{x}, \bar{y} ; \bar{X}, \bar{Y}, \bar{X}+\bar{Y} ; \tilde{x}, \tilde{y}, a \tilde{x}+b \tilde{y})$

$2(x, y, 0 ; X, 0, Y ; 0, \bar{x}, \bar{y} ; \bar{X}, \bar{Y}, \bar{Y} ; \tilde{x}, \tilde{y}, \tilde{x}+b \tilde{y})$
$J^{1} \mathscr{A}$-codim

$2 \quad 1-$ det

2

Case 1. This is a bimodular family and the codimension of the stratum is 0 . We find that the 5-germ is 1 -determined provided $a, b, a-1, b-1, a-b \neq 0$.

Case 2. Provided $b \neq 0,1$, a 2-transversal is $(x, y, 0 ; X, 0, Y ; 0, \bar{x}, \bar{y} ; \bar{X}$, $\left.\bar{Y}, \bar{Y}+a \bar{X}^{2} ; \tilde{x}, \tilde{y}, \tilde{x}+b \tilde{y}\right)$. We take the stratum as $b \neq 0,1$ from now on. Applying Mather's Lemma to the family in $a$ we can reduce the above family to the following two unimodular strata.

$\begin{array}{ccc}\text { 2-jet } & J^{2} \mathscr{A} \text {-cod } & \\ \left(x, y, 0 ; X, 0, Y ; 0, \bar{x}, \bar{y} ; \bar{X}, \bar{Y}, \bar{Y}+\bar{X}^{2} ; \tilde{x}, \tilde{y}, \tilde{x}+b \tilde{y}\right) & 2 & \text { 2-det }(b \neq 0,1) \\ (x, y, 0 ; X, 0, Y ; 0, \bar{x}, \bar{y} ; \bar{X}, \bar{Y}, \bar{Y} ; \tilde{x}, \tilde{y}, \tilde{x}+b \tilde{y}) & 3 & \#\end{array}$

\subsection{Classification of Six-germs}

We need to consider 6-germs with $\mathscr{A}$-codimension $=0$. From Proposition 2.4, the 5-germ formed by the first five branches (say) must be of type (1) in Section 3.4. Ignoring several exceptional cases (which we may do on codimension grounds) we find that the $J^{1} \mathscr{A}$-stratum of 6-germs we need to consider is given by

$$
\left(x, y, 0 ; X, 0, Y ; 0, \bar{x}, \bar{y} ; \bar{X}, \bar{Y}, \bar{X}+\bar{Y} ; \tilde{x}, \tilde{y}, a_{1} \tilde{x}+a_{2} \tilde{y} ; \tilde{X}, \tilde{Y}, a_{3} \tilde{X}+a_{4} \tilde{Y}\right) .
$$

For generic $a_{i}$ a 2-transversal is (spanned by) $\left\{\left(\bar{x}^{2}, 0,0\right),\left(0,0, \tilde{x}^{2}\right),\left(0,0, \tilde{X}^{2}\right)\right\}$. The resulting family is 6 -modular and with a little work can be reduced to the form

$$
\begin{aligned}
& \left(x, y, 0 ; X, 0, Y ; \bar{x}^{2}, \bar{x}, \bar{y} ; \bar{X}, \bar{Y}, \bar{X}+\bar{Y}\right. \\
& \left.\quad \tilde{x}, \tilde{y}, a_{1} \tilde{x}+a_{2} \tilde{y}+a_{3} \tilde{x}^{2} ; \tilde{X}, \tilde{Y}, a_{4} \tilde{X}+a_{5} \tilde{Y}+a_{6} \tilde{X}^{2}\right) .
\end{aligned}
$$


A generic representative of this family has $J^{2} \mathscr{A}$-codimension 6 and the stratum therefore has codimension 0 and contributes to our list of singularities. We do not determine the exceptional values for the moduli in this family. Computer calculations indicate that the defining equations for the corresponding varieties are extremely complicated and likely to be of little practical use. Instead we attempt to show that the family is finitely-determined for generic $a_{i}$. Computer calculations verify that for a fixed value of the moduli (say, $\left(a_{1}, \ldots, a_{6}\right)=(2,3,1,1,5,4)$, chosen following a preliminary investigation of the exceptional values) the 6-germ is 2-determined. (Note, the calculation using fixed moduli is computationally far less intensive.) Since finitedeterminacy is an open condition the conclusion follows. In addition, should we need to consider a specific example in applications then we have a member of the family whose exact determinacy degree (2) is known.

\section{Bifurcation Geometry}

In this section we analyse the bifurcation geometry of the multigerms appearing in Table 2. Versal unfoldings are calculated using the computer package Transversal (unfolding parameters being denoted by $a, a_{i}, b$ and $c$ ). We describe each bifurcation set via its stratification into the five components (denoted by $\mathscr{B}_{1}, \ldots, \mathscr{B}_{5}$ ) corresponding to the $\mathscr{A}_{e}$-codimension 1 degenerations. In many cases this is accompanied by diagrams of the bifurcation set and the transitions which occur in the multigerm as one passes through the bifurcation set. This analysis was carried out in real time using the computer package LSMP [14] but, of course, it is not always possible to reproduce faithful representations of the computer pictures. For example, in some cases it is more informative to show the transitions in the intersection curves of the branches. In addition, such analysis is meaningless for cases where the normal form contains moduli. Here a topological treatment is needed but we do not consider such issues as they would extend the article substantially. We stop at an algebraic description of the bifurcation sets and summarise the types of transitions exhibited; the geometry is notably more complex for the non-simples. The bigerm $A_{0} H_{2}$ and the 6-germ $A_{0}^{6}$ are the only cases we omit completely. The algebraic description of both is extremely complicated (in the former case due to the algebraic form of the double point curve $\left.D^{2}\left(f_{2}\right)\right)$. A more practical approach would be to remove the moduli via topological methods at the outset.

There are five degenerations of $\mathscr{A}_{e}$-codimension 1 , characterised as follows. The strata $\mathscr{B}_{1}, \ldots, \mathscr{B}_{5}$ of the bifurcation set $\mathscr{B}$ are given by taking the closure of the corresponding sets.

$\mathscr{B}_{1}^{ \pm}$fusion/birth of two cross-caps via 'bubbles' $\left(\mathscr{B}_{1}^{+}\right)$or 'cones' $\left(\mathscr{B}_{1}^{-}\right)$. This is a single branch phenomenon which occurs when one branch of the 
multigerm is adjacent to the monogerm $S_{1}^{ \pm}$, [12]. Conditions come directly from the calculations for monogerms carried out in [6].

$\mathscr{B}_{2}^{ \pm}$non-transverse double points: elliptic $\left(\mathscr{B}_{2}^{+}\right)$or hyperbolic $\left(\mathscr{B}_{2}^{-}\right)$. These occur when two smooth branches intersect and have a common tangent plane at the point in question. See $A_{0}^{2} \mid A_{1}^{ \pm}$below.

$\mathscr{B}_{3}$ cross-cap/immersion intersections. These occur when a smooth branch intersects a cross-cap transversally. Precisely (for codimension 1 and not higher degenerations) we require the immersion is transverse to both the cross-cap's tangent line and double point curve, and that we have the least degenerate form of cross-cap, namely one with quadratic double point curve. See $\left(A_{0} S_{0}\right)_{1}$ below (the double point curve being given by $\left.X+Y^{2}=0\right)$.

$\mathscr{B}_{4}$ non-transverse triple points. These occur when two smooth branches intersect transversally, and a third smooth branch makes a tangential intersection with this curve of double points. See $A_{0}^{3} \mid A_{1}$ below.

$\mathscr{B}_{5}$ transverse quadruple points. These occur when four smooth branches intersect. Precisely (for codimension 1 and not higher degenerations) we require each pair of branches to intersect transversally, with the remaining two branches intersecting this curve of double points transversally. See $A_{0}^{4}$ below.

Figure 4.1 shows stable perturbations of these five types close to the bifurcation point.

In the examples below the strata are often given by standard discriminants. For example, discriminants of $x^{k+1}+a_{k-1} x^{k-1}+\cdots+a_{1} x+a_{0}\left(A_{k}\right)$ and discriminants of $x^{k+1}+a_{k} x^{k}+\cdots+a_{1} x+a_{0}$. The latter cases are diffeomorphic to the product of the corresponding $A_{k}$ discriminant with a line; for example, the parabola and the 'twisted-cuspidal-edge surface' for $k=1$ and $k=2$, respectively; see bigerms $A_{0} S_{0} \mid A_{1}^{ \pm}$and $A_{0} S_{0} \mid A_{2}$ and Figures 4.13-4.15. By 'discriminant of $p(x)$ ' we will implicitly mean real discriminant and denote this by $\Delta(p(x))$, that is the hypersurface given by eliminating $x$ from $p(x)=0$ and $d p / d x=0$ for real $x$. For example, with $p(x)=x^{2 k}+a_{k-1} x^{2 k-2}+$ $\cdots a_{1} x^{2}+a_{0}$ this imposes further restrictions and gives the 'half-parabola' $\left(a_{1}^{2}-4 a_{0}=0, a_{1} \leq 0\right)$ and the 'half twisted-cuspidal-edge' for $k=2$ and $k=3$, respectively. See bigerms $\left(A_{0} S_{0}\right)_{2}$ and $\left(A_{0} S_{0}\right)_{3}$ and Figures 4.8 and 4.9.

It is informative to calculate the bifurcation sets for general series of simple singularities where possible. As the calculations are routine we give the proofs in several cases and state the remaining ones. Table 3 summarises these results for all of the simple singularities appearing in Table 2. The remaining nonsimples are discussed as individual cases below. 


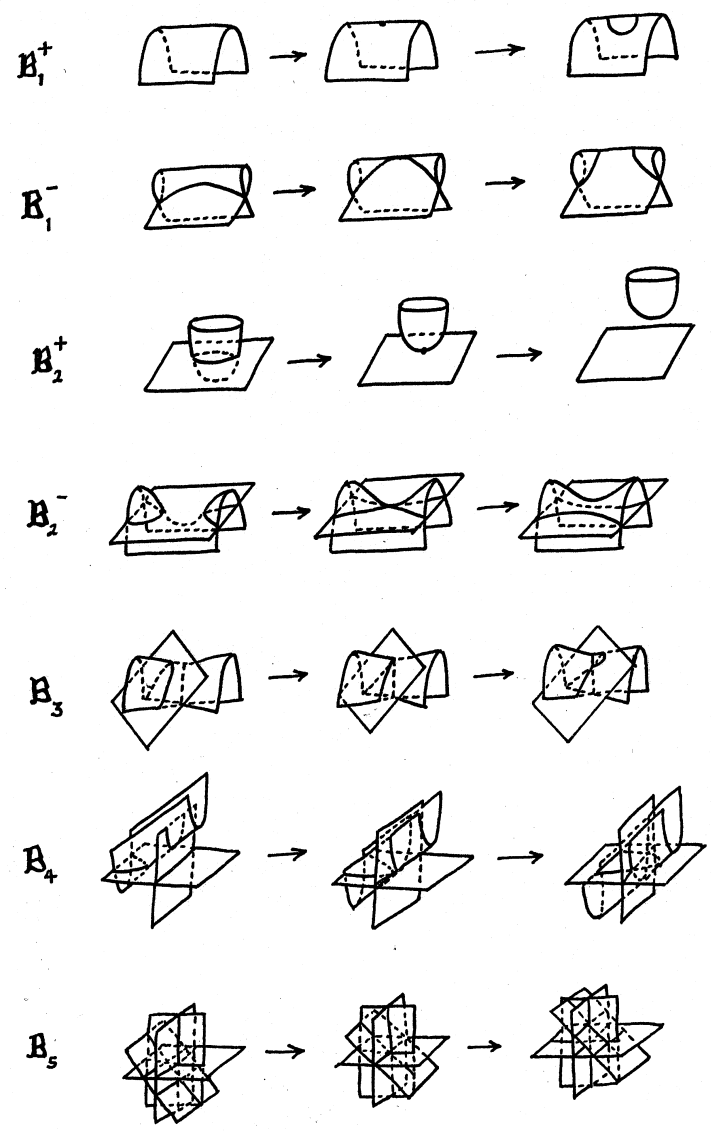

FIG. 4.1

\subsection{Bifurcation of the Simple Bigerms}

Series $A_{0}^{2} \mid A_{k}^{ \pm}$. This represents the intersection of two immersed sheets. An $\mathscr{A}_{e}$-versal unfolding is given by $\left(f_{1} ; f_{2}\right)=(x, y, 0 ; X, Y, p(X, Y))$ where $p(X, Y)=X^{2} \pm Y^{k+1}+a_{k-1} Y^{k-1}+\cdots+a_{1} Y+a_{0}$ defines the intersection curve of $f_{1}$ and $f_{2}$ and their contact type. The only non-empty stratum of $\mathscr{B}$ is $\mathscr{B}_{2}$ which is given by the discriminant of $p(0, Y)$.

The proof is similar to that of case $\left(A_{0} S_{0}\right)_{k}$, which we take as as our main example. The bigerms $A_{0}^{2} \mid A_{1}^{ \pm}$are the archetypes of the non-transverse elliptic and hyperbolic double points, respectively types $\mathscr{B}_{2}^{+}$, and $\mathscr{B}_{2}^{-}$. One may distinguish $\mathscr{B}_{2}^{+}$and $\mathscr{B}_{2}^{-}$points geometrically by the intersection curve of $f_{1}$ and $f_{2}$ as this identifies the order of contact of the branches. Note that $p$ is a versal 


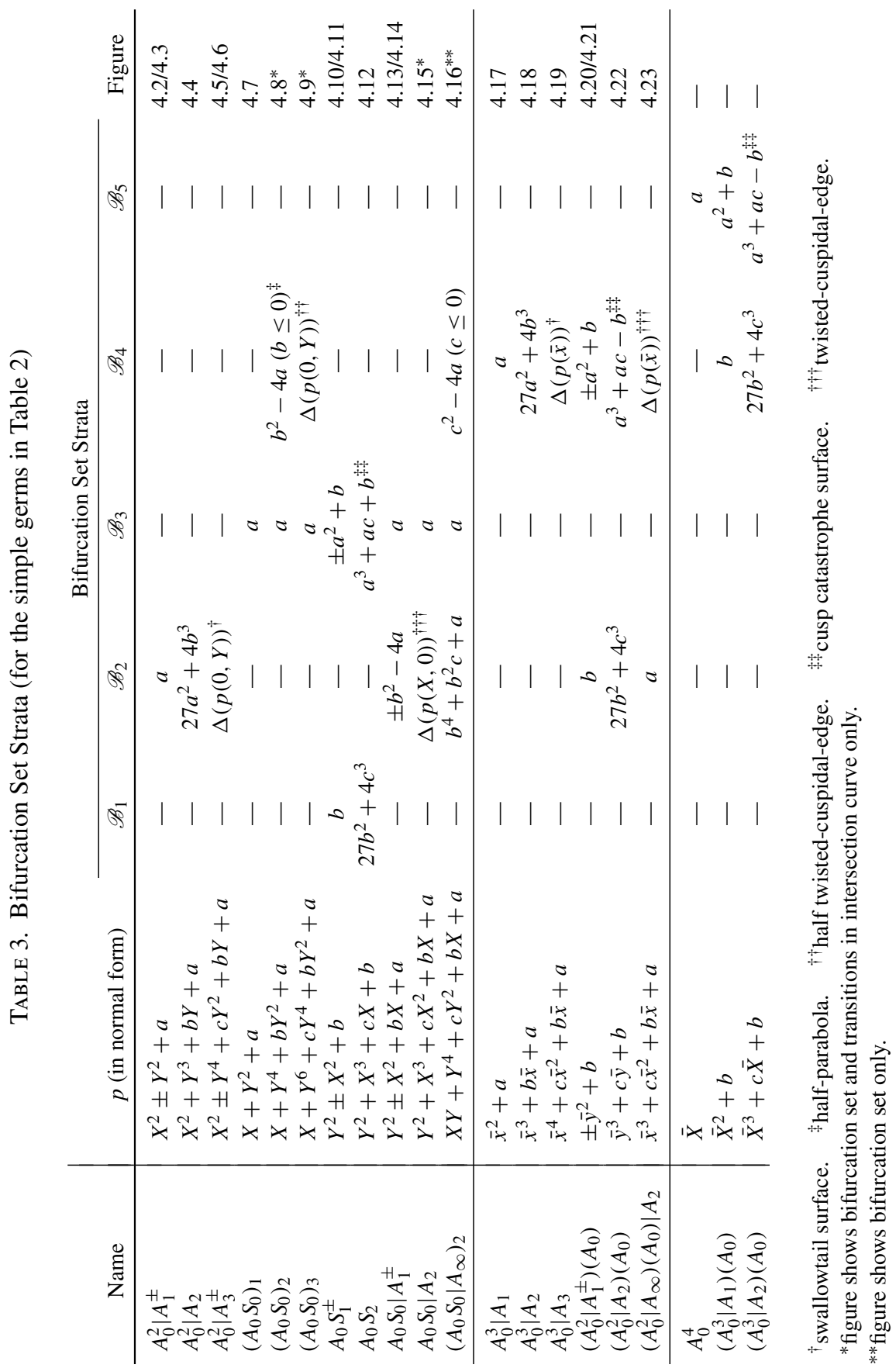


unfolding of the intersection curve, which in the general case is of type $A_{k}$, as suggested by the computer pictures.

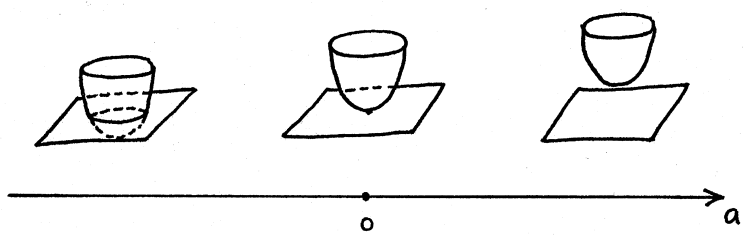

FIG. $4.2 A_{0}^{2} \mid A_{1}^{+}$

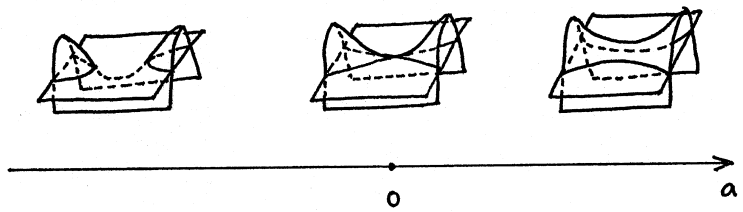

FIG. $4.3 A_{0}^{2} \mid A_{1}^{-}$

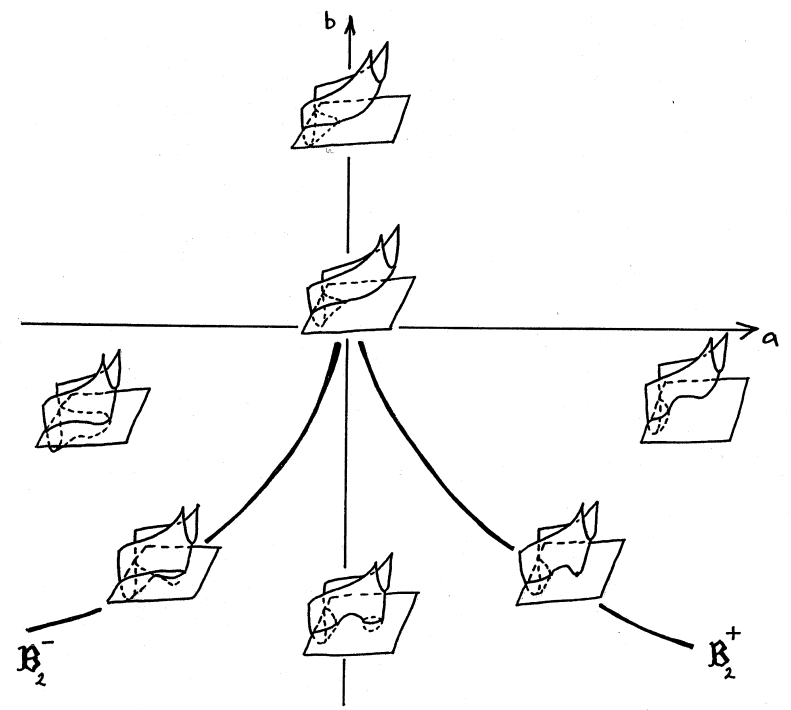

FIG. $4.4 A_{0}^{2} \mid A_{2}$ 


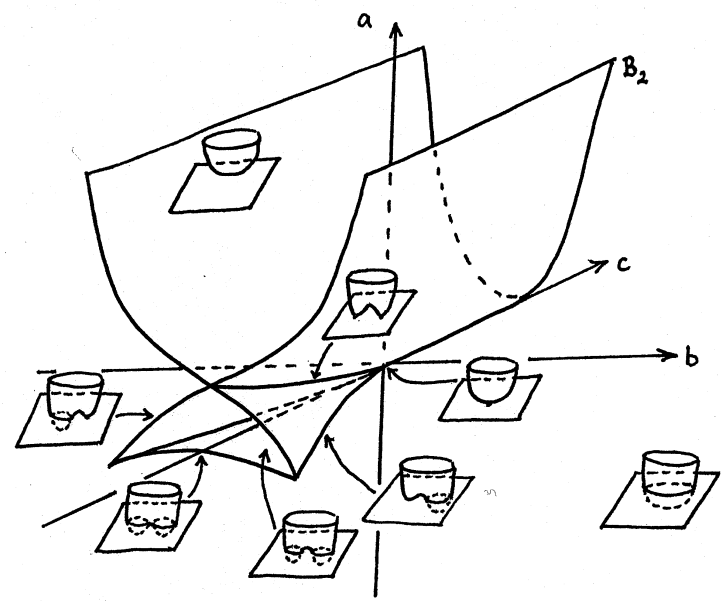

FIG. $4.5 A_{0}^{2} \mid A_{3}^{+}$

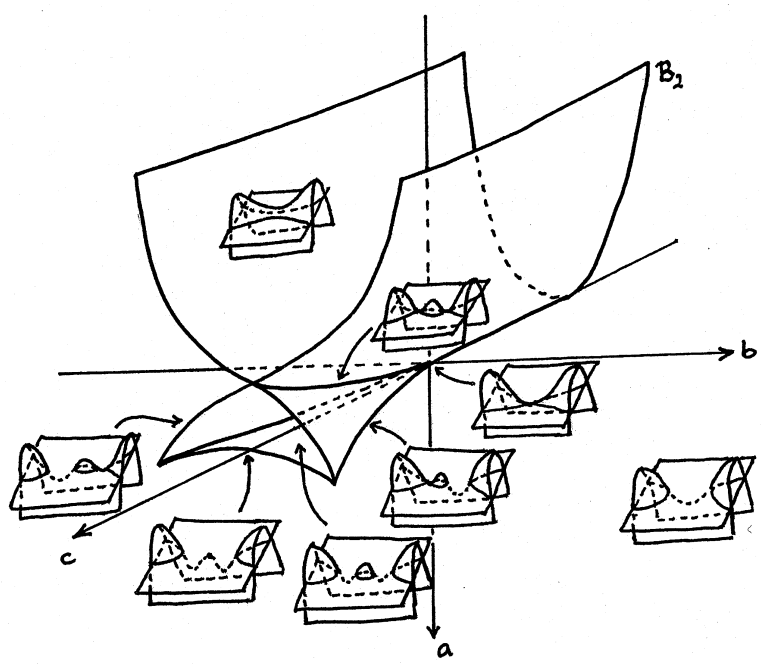

FIG. $4.6 A_{0}^{2} \mid A_{3}^{-}$

Series $\left(A_{0} S_{0}\right)_{k}$. This represents the transverse intersection of a sheet and a cross-cap. An $\mathscr{A}_{e}$-versal unfolding is given by $\left(f_{1} ; f_{2}\right)=\left(x, y, 0 ; Y^{2}\right.$, $Y p(X, Y), X)$ where $p(X, Y)=X+Y^{2 k}+a_{k-1} Y^{2 k-2}+\cdots+a_{1} Y^{2}+a_{0}$ defines $D^{2}\left(f_{2}\right)$. The non-empty strata of $\mathscr{B}$ are $\mathscr{B}_{3}$ and $\mathscr{B}_{4}$, given by $a_{0}=0$ and the discriminant of $p(0, Y)$, respectively. 
Proof. The versal unfolding is calculated using the standard unfolding theorem extended to the multigerm case. The details are routine and omitted. We will need to consider the multilocal behaviour of each branch, in particular determine its double point set in the source. The following remarks are useful in many other cases as well. Given a monogerm $f: \mathbf{R}^{2}, 0 \rightarrow \mathbf{R}^{3}, 0$ we define the source double point set $D^{2}(f)$ to be the closure of the set $\left(x_{1}, y_{1}\right) \in \mathrm{R}^{2}$ such that there exists $\left(x_{2}, y_{2}\right) \in \mathrm{R}^{2}$ with $\left(x_{1}, y_{1}\right) \neq\left(x_{2}, y_{2}\right)$ and $f\left(x_{1}, y_{1}\right)=f\left(x_{2}, y_{2}\right)$. (Strictly speaking, $D^{2}(f)$ is a set germ defined via representatives of the germ $f$.) Given a monogerm $f$ with 2 -jet $j^{2} f=\left(x, y^{2}, 0\right)$ we can write $f$ in the form $\left(x, y^{2}, y p(x, y)\right)$ where $p$ is even in $y$, that is $p(x, y)=\tilde{p}\left(x, y^{2}\right)$ for some $\tilde{p}$; see [12]. In this case we can calculate $D^{2}(f)$ from first principles: we require $x_{1}=x_{2}, y_{1}=-y_{2} \neq 0$ and $p\left(x_{1}, y_{1}\right)=0$. Taking the closure of such $\left\{\left(x_{1}, y_{1}\right)\right\}$ we see that $D^{2}(f)$ is given by $p(x, y)=0$. This applies to many of the cases we have to consider and can be extended to the other cases using similar arguments.

$\mathscr{B}_{1}$ stratum: since neither branch is more degenerate than a cross-cap it cannot deform (be adjacent) to an $S_{1}$ singularity. The $\mathscr{B}_{1}$ stratum is therefore empty.

$\mathscr{B}_{2}$ stratum: the two branches intersect when $X=0$. However, since

$$
d f_{2}=\left(\begin{array}{cc}
0 & 2 Y \\
Y \partial p / \partial X & p+Y \partial p / \partial Y \\
1 & 0
\end{array}\right)
$$

one sees that the two branches are never tangential. Likewise one sees that a point $\left(X_{1}, Y_{1}\right)$ on $D^{2}\left(f_{2}\right)$ and its corresponding point $\left(X_{1},-Y_{1}\right)$ never give rise to a tangential intersection in the image. Thus $\mathscr{B}_{2}$ is empty.

$\mathscr{B}_{3}$ stratum: this type of degeneracy (or worse) can only occur when both branches intersect and $f_{2}$ has a cross-cap at the intersection point. Respectively, $X=0$ and, from the expression for $d f_{2}: Y=0, p(X, Y)=0$. That is, $p(0,0)=0$ and $\mathscr{B}_{3}$ is just $\left\{a_{0}=0\right\}$.

$\mathscr{B}_{4}$ stratum: triple points can only occur when the image of $f_{1}$ intersects the image of $D^{2}\left(f_{2}\right)$. Now $f_{1}$ intersects $f_{2}$ when $X=0 . D^{2}\left(f_{2}\right)$ is given by $p(X, Y)=0$ with tangent space (at a given point $(X, Y)$ ) given by the kernel of $d p$. This is spanned by $(-\partial p / \partial Y, 1)$ and so the tangent to the intersection curve in the target is spanned by the image of this vector under $d f_{2}$. Therefore $f_{2}\left(D^{2}\right)$ intersects $f_{1}$ tangentially precisely when $X=0$ and $p=\partial p / \partial Y=0$. Thus $\mathscr{B}_{4}$ is given by the discriminant of $p(0, Y)$.

$\mathscr{B}_{5}$ stratum: since there are no quadruple points this stratum is empty.

The bigerm $\left(A_{0} S_{0}\right)_{1}$ is the archetype of the degeneration type $\mathscr{B}_{3}$. Note that in the calculation of $\mathscr{B}_{4}=\Delta(p(0, Y))$ we can assume that $Y \neq 0$ so that 
$(X, Y)$ is a proper double point (otherwise $(X, Y)$ is a cross-cap point and the condition for instability is $a_{0}=0$, as found for the $\mathscr{B}_{3}$ stratum) allowing us to eliminate $Y$. Thus, for $\left(A_{0} S_{0}\right)_{2}, \mathscr{B}_{4}$ is the half-parabola $\left\{(a, b): a_{1}^{2}-4 a_{0}=\right.$ $\left.0, a_{1} \leq 0\right\}$, and for $\left(A_{0} S_{0}\right)_{3}$ it is the half twisted-cuspidal-edge. Note that the intersection curve of $f_{1}$ and $f_{2}$ in the target is given parametrically as the plane curve $\left(Y^{2}, Y p(0, Y), 0\right)$, a versal unfolding of the planar cusp $\left(t^{2}, t^{2 k+1}\right)$. The bifurcation geometry of $\left(A_{0} S_{0}\right)_{1},\left(A_{0} S_{0}\right)_{2}$ and $\left(A_{0} S_{0}\right)_{3}$ therefore coincides with, respectively, that of the cusp $\left(t^{2}, t^{3}\right)$, rhamphoid cusp $\left(t^{2}, t^{5}\right)$ and higher rhamphoid cusp $\left(t^{2}, t^{7}\right)$, as suggested by the computer pictures.
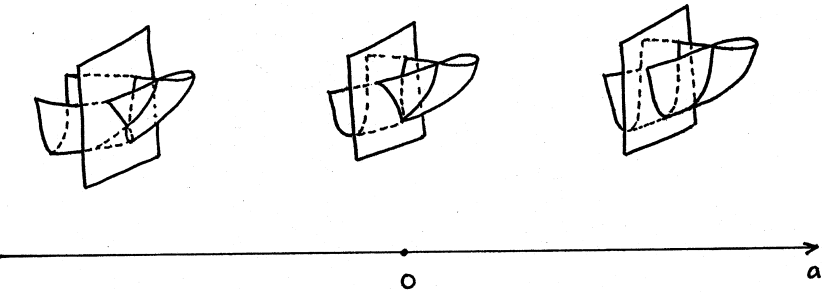

FIG. $4.7\left(A_{0} \mid S_{0}\right)_{1}$

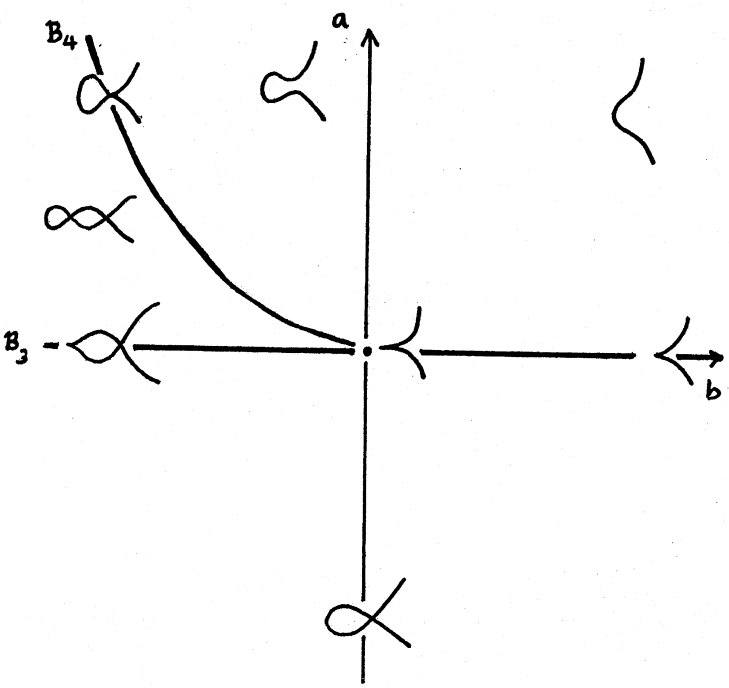

FIG. $4.8\left(A_{0} \mid S_{0}\right)_{2}$ 


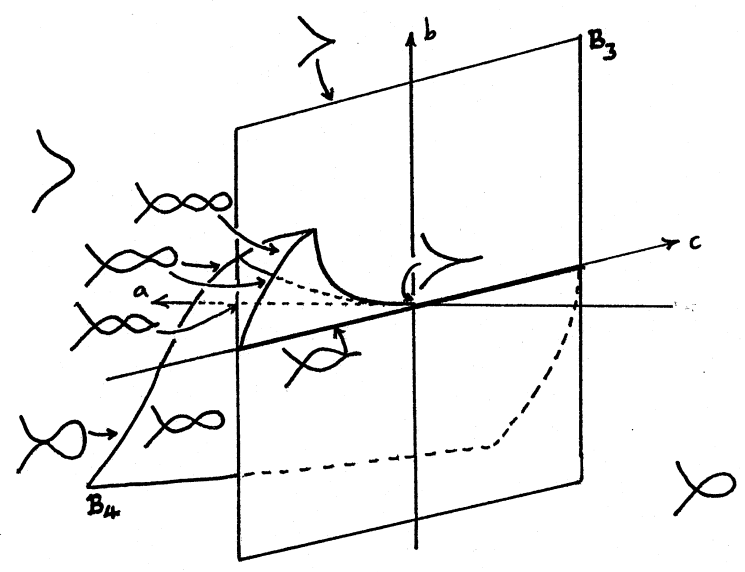

FIG. $4.9\left(A_{0} \mid S_{0}\right)_{3}$

Series $A_{0} S_{k}^{ \pm}$. An $\mathscr{A}_{e}$-versal unfolding is $\left(f_{1} ; f_{2}\right)=(x, y, a ; Y p(X, Y)$, $\left.Y^{2}, X\right)$ where $p(X, Y)=Y^{2} \pm X^{k+1}+b_{k-1} X^{k-1}+\cdots+b_{1} X+b_{0}$ defines $D^{2}\left(f_{2}\right)$. The non-empty strata of $\mathscr{B}$ are $\mathscr{B}_{1}$ and $\mathscr{B}_{3}$, given by the discriminant of $p(X, 0)$ and $p(a, 0)=0$, respectively.

Proof. The proof follows similar arguments to that of case $\left(A_{0} S_{0}\right)_{k}$. We will just remark on the following. The $\mathscr{B}_{3}$ and $\mathscr{B}_{4}$ strata are calculated as before. For the $\mathscr{B}_{3}$ stratum: the intersection condition is $X=a$; the conditions for $f_{2}$ to be singular are $Y=0, p(X, Y)=0 . \mathscr{B}_{4}$ stratum: again the intersection condition is $X=a ; D^{2}\left(f_{2}\right)$ is given by $p(X, Y)=0$ with the tangency condition being $Y=0$. However, proper double points are given by $p(X, Y)=0$ with $Y \neq 0$, whereas the isolated double points with $Y=0$ have multiplicity 2 and coincide with cross-caps points. (This behaviour was noted above.) Thus, $\mathscr{B}_{3}$ is given by $p(a, 0)=0$ while $\mathscr{B}_{4}$ is empty. These observations provide a convenient method for calculating the $\mathscr{B}_{1}$ stratum.

$\mathscr{B}_{1}$ stratum: here $f_{2}$ is more degenerate than a cross-cap so we should expect it to deform to an $S_{1}$ singularity. Cross-cap points (in fact, any singular points) are given by $Y=0, p(X, Y)=0$. Such points therefore lie on the double point curve $D^{2}\left(f_{2}\right)$ and coalesce to form an $S_{1}$ point when $p(X, 0)$ has a repeated root. Thus, $\mathscr{B}_{1}$ is given by the discriminant of $p(X, 0)$.

Note that the intersection curve of $f_{1}$ and $f_{2}$ is given parametrically as a versal unfolding of the standard planar cusp $\left(t^{2}, t^{3}\right)$. For example, the bifurcation set for $A_{0} S_{2}$ consists of a cuspidal edge meeting the cusp catastrophe surface tangentially. Points on the cusp catastrophe surface correspond to a cross-cap point (which appears in the unfolding of $S_{2}$ ) intersecting a sheet, 
and in this situation the two branches $f_{1}, f_{2}$ always meet in a cusp. Inside the cuspidal edge $S_{2}$ deforms into three cross-cap points so there are three occasions where the sheet $f_{1}$ can meet $f_{2}$ in a cusp as one varies $a$. Whereas outside the cuspidal edge only one cross-cap appears and there is only one occasion where $f_{1}$ intersects $f_{2}$ in a cusp.

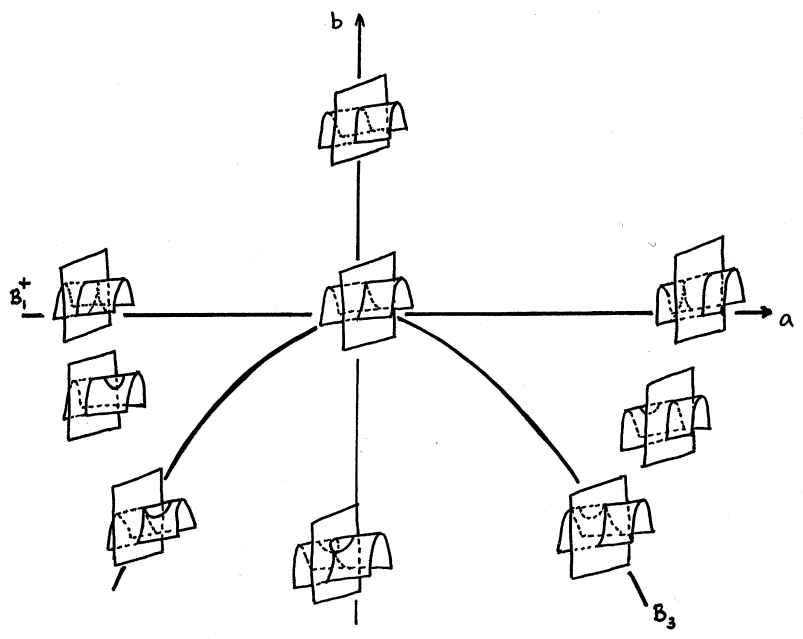

FIG. $4.10 A_{0} S_{1}^{+}$

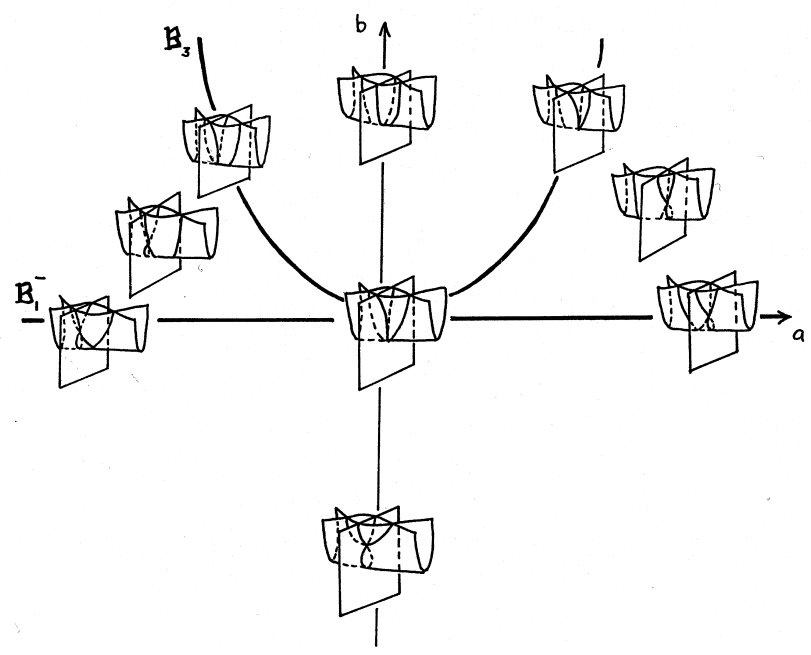

FIG. $4.11 A_{0} S_{1}^{-}$ 


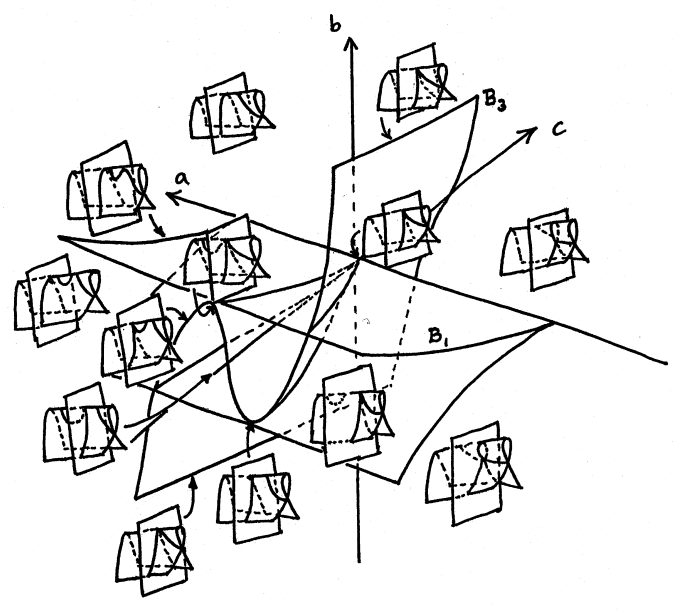

FIG. $4.12 A_{0} S_{2}$

Series $A_{0} S_{0} \mid A_{k}^{ \pm}$. This represents a sheet and cross-cap intersecting tangentially. An $\mathscr{A}_{e}$-versal unfolding is given by $\left(f_{1} ; f_{2}\right)=(x, y, 0 ; X, X Y, p(X, Y))$ where $p(X, Y)=Y^{2} \pm X^{k+1}+a_{k} X^{k}+\cdots+a_{1} X+a_{0}$. The non-empty strata of $\mathscr{B}$ are $\mathscr{B}_{2}$ and $\mathscr{B}_{3}$, given by the discriminant of $p(X, 0)$ and $a_{0}=0$, respectively.

Proof. The proof follows similar arguments to that of case $\left(A_{0} S_{0}\right)_{k}$. The same argument shows that $D^{2}\left(f_{2}\right)$ is given by $X=0$, with $Y \neq 0$ required for proper double points.

With a little work one can show that the intersection curve of the branches is given by $y^{2} \pm x^{k+3}+a_{k} x^{k+2}+\cdots+a_{1} x^{3}+a_{0} x^{2}=0$ (minus the origin in the case where it is an isolated point). This is a (non-versal) unfolding of an $A_{k+2}$ singularity and exhibits the expected transitions. (In the case $k=2$ we only show the intersection curve, and do so for sections $\{c=$ constant $\}$ of the cuspidal edge for clarity.)

Series $\left(A_{0} S_{0} \mid A_{\infty}\right)_{k}$. This represents the most degenerate type of sheet/crosscap intersection, where the sheet is tangential to both the cross-cap and its double point curve. An $\mathscr{A}_{e}$-versal unfolding is given by $\left(f_{1} ; f_{2}\right)=(x, y, 0 ; X$, $\left.Y^{2}, p(X, Y)\right)$ where $p(X, Y)=X Y+Y^{2 k}+a_{k-1} Y^{2 k-2}+\cdots+a_{1} Y^{2}+a_{0}+b X$. The non-empty strata of $\mathscr{B}$ are $\mathscr{B}_{2}, \mathscr{B}_{3}$ and $\mathscr{B}_{4}$, given by $p(0, b)=0, a_{0}=0$ and the discriminant of $p(0, Y)$, respectively.

We remark that $D^{2}\left(f_{2}\right)$ is again given by $X=0$. The geometry for type $\left(A_{0} S_{0} \mid A_{\infty}\right)_{2}$ is complicated and we do not try to depict the transitions. The bifurcation set consists of a higher degree version of the cusp catastrophe sur- 
face, a plane, and a half-parabola edge, corresponding to the three components $\mathscr{B}_{2}, \mathscr{B}_{3}$ and $\mathscr{B}_{4}$ respectively.

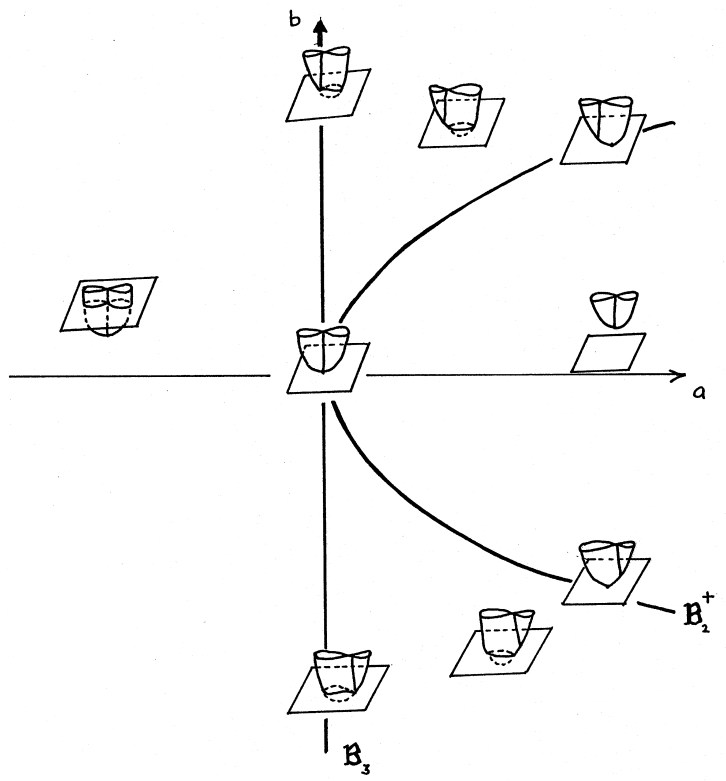

FIG. $4.13 A_{0} S_{0} \mid A_{1}^{+}$

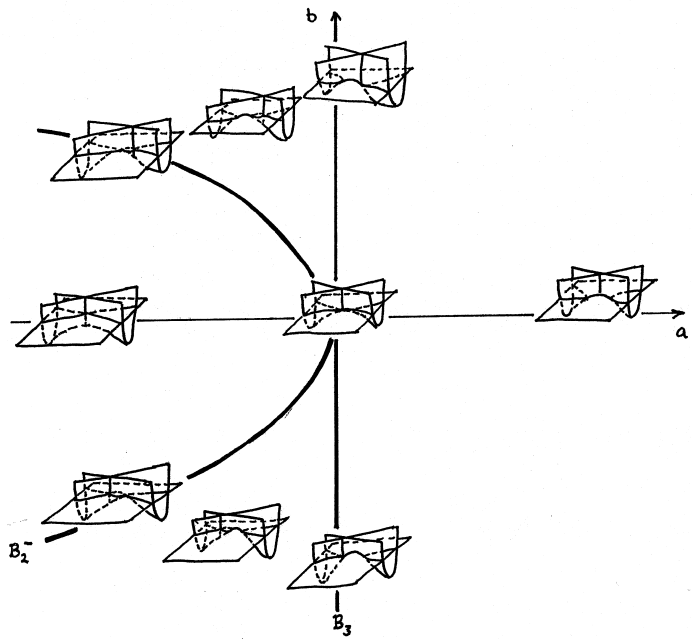

FIG. $4.14 A_{0} S_{0} \mid A_{1}^{-}$ 


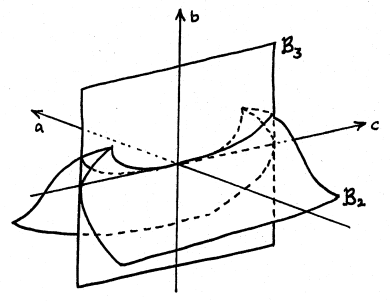

Bifurcation Set
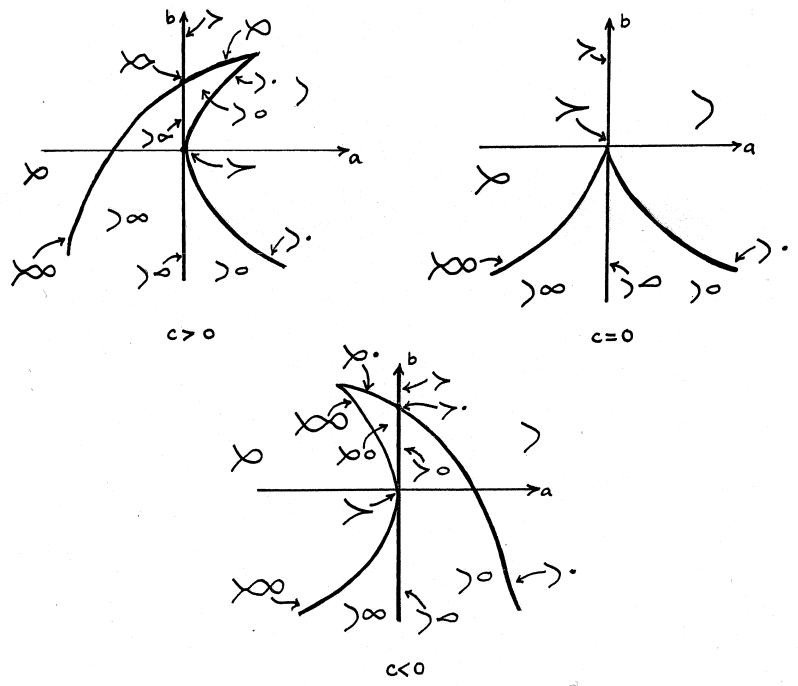

FIG. $4.15 A_{0} S_{0} \mid A_{2}$

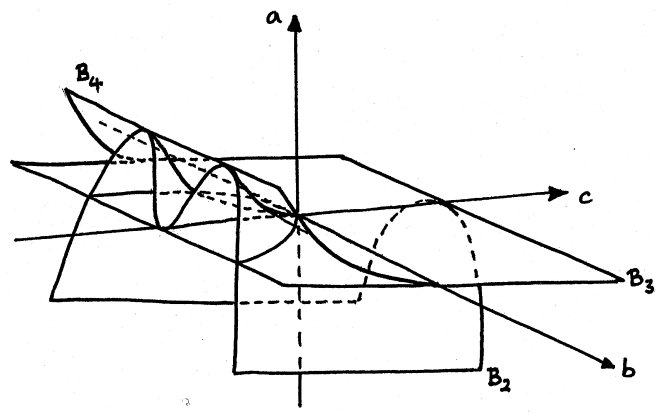

FIG. $4.16\left(A_{0} S_{0} \mid A_{\infty}\right)_{2}$ 


\subsection{Bifurcation of the Non-simple Bigerms}

For each case we can incorporate the modulus $a$ as an unfolding parameter. We use the notation $\bar{a}$ to highlight the fact that $\bar{a}$ is a modulus in the original germ.

Type $A_{0} B_{2}$. An $\mathscr{A}_{e}$-versal unfolding is $\left(f_{1} ; f_{2}\right)=\left(x, y, 0 ; Y p(X, Y), Y^{2}\right.$, $X)$ where $p(X, Y)=X^{2}+X Y^{2}+\bar{a} Y^{4}+b Y^{2}+c X+d$ defines $D^{2}\left(f_{2}\right)$ and $\bar{a} \neq 0, \frac{1}{4}$. Singular points of $f_{2}$ are given by $Y=p(X, Y)=0$, so $\mathscr{B}_{1}$ points are characterised by the requirement that such points on $D^{2}\left(f_{2}\right)$ coalesce, the strata being defined by $\Delta(p(X, 0))$, that is $c^{2}-4 d=0$. Although $f_{1}$ and $f_{2}$ are never tangential we can obtain non-transverse double points from a point $(X, Y)$ on $D^{2}\left(f_{2}\right)$ and its partner $(X,-Y)$. This behaviour has not been exhibited thus far. Such adjacencies are noted in the work of Mond [12], where it is stated that the $B_{k}$ series $\left(x, y^{2}, x^{2} y \pm y^{2 k+1}\right)$ is adjacent to the bigerm $A_{0}^{2} \mid A_{k-1}^{ \pm}$. In the present scenario $f_{2}$ is $\mathscr{A}$-equivalent to $B_{2}$ and the adjacency with $A_{0}^{2} \mid A_{1}^{ \pm}$provides $\mathscr{B}_{2}$ type tangencies. It turns out that the $\mathscr{B}_{2}$ stratum is given by eliminating $X$ and $Y$ from $p=\partial p / \partial X=\partial p / \partial Y=0$, giving the hypersurface $(2 b-c)^{2}+(1-4 \bar{a})\left(4 d-c^{2}\right)=0$ with the constraint $(1-4 \bar{a})(2 b-c) \geq 0 . \mathscr{B}_{3}$ points occur when $f_{1}$ meets a singular point of $f_{2}$. The requirement is simply $p(0,0)=0$ so the stratum is given by $d=0$. $\mathscr{B}_{4}$ points occur when $f_{1}$ intersects $f_{2}\left(D^{2}\left(f_{2}\right)\right)$ tangentially, the stratum is found to be given by $\Delta(p(0, Y))$, that is $4 \bar{a} d-b^{2}=0$ with $\bar{a} b \leq 0$. Finally, $\mathscr{B}_{5}$ is empty.

Type $A_{0} S_{1} \mid A_{1}^{ \pm}$. An $\mathscr{A}_{e}$-versal unfolding is $\left(f_{1} ; f_{2}\right)=(x, y, 0 ; X, Y p(X, Y)$, $\left.Y^{2} \pm X^{2}+d\right)$ where $p(X, Y)=Y^{2}+\bar{a} X^{2}+b X+c$ defines $D^{2}\left(f_{2}\right)$ and $\bar{a} \neq 0, \pm 1$. Singular points of $f_{2}$ are given by $Y=p(X, Y)=0$, so $\mathscr{B}_{1}$ is defined by $\Delta(p(X, 0))$, that is $b^{2}-4 \bar{a} c=0 . \mathscr{B}_{2}$ points occur when $f_{1}$ and $f_{2}$ are tangential, the stratum is shown to be given by $d=0$. Combining the conditions for $f_{1}$ to meet a singular point of $f_{2}$, we find that $\mathscr{B}_{3}$ is given by eliminating $X$ from $\pm X^{2}+d=\bar{a} X^{2}+b X+c=0$, giving $\bar{a}^{2} d^{2}+c^{2} \pm$ $\left(b^{2} d-2 \bar{a} c d\right)=0$ with $b^{2}-4 \bar{a} c \geq 0$ and $d \leq 0$ ( + case $), d \geq 0$ ( - case) . $\mathscr{B}_{4}$ points occur when $f_{1}$ meets $f_{2}\left(D^{2}\left(f_{2}\right)\right)$ tangentially, the stratum is found to be $b^{2}+4(c-d)( \pm 1-\bar{a})=0 . \mathscr{B}_{5}$ is empty.

Type $S_{0} S_{0}$. An $\mathscr{A}_{e}$-versal unfolding is $\left(f_{1} ; f_{2}\right)=\left(x, y p(x, y), y^{2} ; Y q(X, Y)\right.$, $\left.X, Y^{2}+d\right)$ where $p(x, y)=x+y^{2}+b$ defines $D^{2}\left(f_{1}\right), q(X, Y)=X+\bar{a} Y^{2}+c$ defines $D^{2}\left(f_{2}\right)$ and $\bar{a} \neq 0$. Neither branch is more degenerate than a cross-cap so $\mathscr{B}_{1}$ is empty. $\mathscr{B}_{2}$ points occur when $f_{1}$ and $f_{2}$ are tangential and a little work shows that the stratum is $d=0 . \mathscr{B}_{3}$ has two components given by $f_{2}$ meeting a singular point of $f_{1}$, and vice-versa. These components are given by eliminating $Y$ from $\bar{a} Y^{3}+c Y+b=Y^{2}+d=0$ giving $b^{2}+d(c-\bar{a} d)^{2}=0$, and 
$y$ from $y^{3}+b y+c=y^{2}-d=0$ giving $c^{2}-d(b+d)^{2}=0$, respectively. $\mathscr{B}_{4}$ has two components given by $f_{2}$ meeting $f_{1}\left(D^{2}\left(f_{1}\right)\right)$ tangentially, and vice-versa. These are both unimodular families of 'twisted-cuspidal-edge surfaces' given by the discriminants of the polynomials $\bar{a} Y^{3}+Y^{2}+c Y+d$ and $y^{3}+\bar{a} y^{2}+b y+$ $c-\bar{a} d$. Another feature with this example is that a point $(x, y) \in D^{2}\left(f_{1}\right)$ can meet a point $(X, Y) \in D^{2}\left(f_{2}\right)$ in the target which, together with their partners, gives rise to a quadruple point in the target space. This bigerm is found to have non-empty $\mathscr{B}_{5}$ stratum given by $\bar{a} b-\bar{a} d-c=0$ with $b, \bar{a} c \leq 0$.

\subsection{Bifurcation of Trigerms}

The calculations are again routine and the case $\left(A_{0} S_{0} \mid A_{1}^{ \pm}\right)\left(A_{0}\right)_{1}$ serves as our main example. We discuss this next and then summarise the remaining cases.

Type $\left(A_{0} S_{0} \mid A_{1}^{ \pm}\right)\left(A_{0}\right)_{1}$. This trigerm consists of two immersions and a cross-cap. An $\mathscr{A}_{e}$-versal unfolding is given by $\left(f_{1} ; f_{2} ; f_{3}\right)=(x, y, 0 ; X, 0, Y$; $\left.\bar{y} p(\bar{x}, \bar{y}), \bar{x}, \bar{y}^{2} \pm \bar{x}^{2}+c \bar{x}+b\right)$ where $p(\bar{x}, \bar{y})=\bar{x}+\bar{y}^{2}+a$ defines $D^{2}\left(f_{3}\right)$. The non-empty strata of $\mathscr{B}$ are $\mathscr{B}_{2}$ which is given by $c^{2} \mp 4 b=0, \mathscr{B}_{3}$ which has two components given by $a\left( \pm a^{2}-a c+b\right)=0, \mathscr{B}_{4}$ given by $b=0$, and $\mathscr{B}_{5}$ given by $a-b=0$ with $a \leq 0$. It is a simple exercise to sketch the bifurcation set or reproduce it on a computer. However, it has five separate components and the resulting picture is not particularly easy to interpret. We will encounter similar problems with some of the other higher multigerms and in such cases will omit the corresponding diagrams.

Proof. The versal unfolding calculation is routine and the fact that $p$ defines $D^{2}\left(f_{3}\right)$ follows from the arguments in case $\left(A_{0} S_{0}\right)_{k}$.

$\mathscr{B}_{1}$ stratum: since no branch is more degenerate than a cross-cap the $\mathscr{B}_{1}$ stratum is empty.

$\mathscr{B}_{2}$ stratum: one sees that only $f_{1}$ and $f_{3}$ can form a tangential intersection. The condition for intersection is $\bar{y}^{2} \pm \bar{x}^{2}+c \bar{x}+b=0$. Now

$$
d f_{3}=\left(\begin{array}{cc}
\bar{y} & \bar{x}+3 \bar{y}^{2}+a \\
1 & 0 \\
\pm 2 \bar{x}+c & 2 \bar{y}
\end{array}\right)
$$

so $f_{1}$ and $f_{3}$ intersect tangentially if, in addition, $\pm 2 \bar{x}+c=0$ and $\bar{y}=0 . \mathscr{B}_{2}$ is therefore given by the discriminant of $\pm \bar{x}^{2}+c \bar{x}+b$.

$\mathscr{B}_{3}$ stratum: this type of degeneracy (or worse) occurs when $f_{1}$ or $f_{2}$ intersects a singular point of $f_{3}$. The latter is given by $\bar{y}=0, \bar{x}=-a$. Combining with the respective intersection conditions, $\bar{y}^{2} \pm \bar{x}^{2}+c \bar{x}+b=0$ and $\bar{x}=0$, we obtain the corresponding two components of the strata.

$\mathscr{B}_{4}$ stratum: triple points occur when all three branches intersect or when one of the immersed branches meets a point of $D^{2}\left(f_{3}\right)$ in the target. This gives three 
possible components to $\mathscr{B}_{4}$ which we will consider in turn. (i): $f_{1}$ and $f_{2}$ meet in the target in the $u$-axis. $f_{3}$ intersects this curve when $\bar{x}=0$ and $\bar{y}^{2}+b=0$ and tangency is a linear dependence condition on the vectors formed by the columns of $d f_{3}$ and $(1,0,0)$, that is $\bar{y}=0$. The resulting component is therefore given by $b=0$. (ii): $f_{1}$ intersects $f_{3}$ when $\bar{y}^{2} \pm \bar{x}^{2}+c \bar{x}+b=0 . D^{2}\left(f_{3}\right)$ is given by $p(\bar{x}, \bar{y})=0$ with tangent space (at a given point $(\bar{x}, \bar{y})$ ) given by the kernel of $d p$. This is spanned by $(-2 \bar{y}, 1)$ and the required tangency condition, that $d f_{3}(\operatorname{Ker} d p)$ lies in the $(u, v)$-plane, comes out as $\pm 2 \bar{x} \bar{y}+c \bar{y}-\bar{y}=0$. For proper $D^{2}$ points $\bar{y} \neq 0$ and this reduces to $\bar{x}= \pm(1-c) / 2$. However, we are interested in semilocal phenomena, that is for all $(a, b, c) \in \mathscr{B}$ in a sufficiently small neighbourhood of the origin, the corresponding points $(\bar{x}, \bar{y})$ must lie in a given (albeit arbitrary) neighbourhood of $(0,0)$. This is clearly not the case so we can rule out the above behaviour and this component of the stratum is empty. (iii): similarly the intersection condition for $f_{2}$ and $f_{3}$ is $\bar{x}=0$ with tangency condition $\bar{y}=0$. However, such points on $D^{2}\left(f_{3}\right)$ are not proper, but cross-cap points. (Indeed, substituting these conditions into $p(\bar{x}, \bar{y})=0$ gives $a=0$, which is one component of the $\mathscr{B}_{3}$ stratum found above.) This component of the strata is therefore empty.

$\mathscr{B}_{5}$ stratum: quadruple points occur when $f_{1}$ and $f_{2}$ intersect a point of $D^{2}\left(f_{3}\right)$ in the target. As already stated, all three branches meet when $\bar{x}=0$ and $\bar{y}^{2}+b=0$. The condition for $(\bar{x}, \bar{y})$ to lie on $D^{2}\left(f_{3}\right)$ then becomes $\bar{y}^{2}+a=0$ and $\mathscr{B}_{5}$ is given by $a=b$, both being non-positive.

Series $A_{0}^{3} \mid A_{k}$. An $\mathscr{A}_{e}$-versal unfolding is given by $\left(f_{1} ; f_{2} ; f_{3}\right)=(x, y, 0$; $X, 0, Y ; \bar{x}, \bar{y}, \bar{y}+p(\bar{x}))$ where $p(\bar{x})=\bar{x}^{k+1}+a_{k-1} \bar{x}^{k-1}+\cdots+a_{1} \bar{x}+a_{0}$. The only non-empty stratum of $\mathscr{B}$ is $\mathscr{B}_{4}$ which is given by the discriminant of $p(\bar{x})$.

The trigerm $A_{0}^{3} \mid A_{1}$ is the archetype of the non-transverse triple point, type $\mathscr{B}_{4}$. Note that $p$ is versally unfolded. Thus for trigerm $A_{0}^{3} \mid A_{2}, \mathscr{B}_{4}$ is given by the $A_{2}$ cusp discriminant; observe that for $(a, b)$ inside the cusp the trigerm has two 'bubbles', which degenerate to one bubble on the bifurcation set and then disappear.
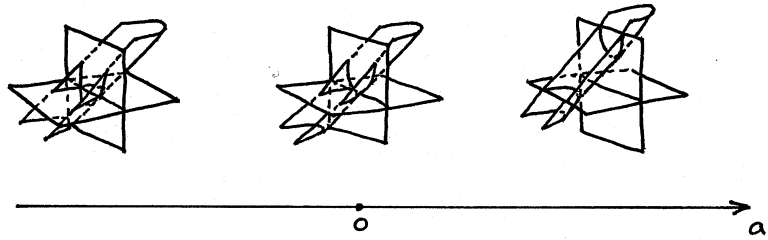

FIG. $4.17 A_{0}^{3} \mid A_{1}$ 


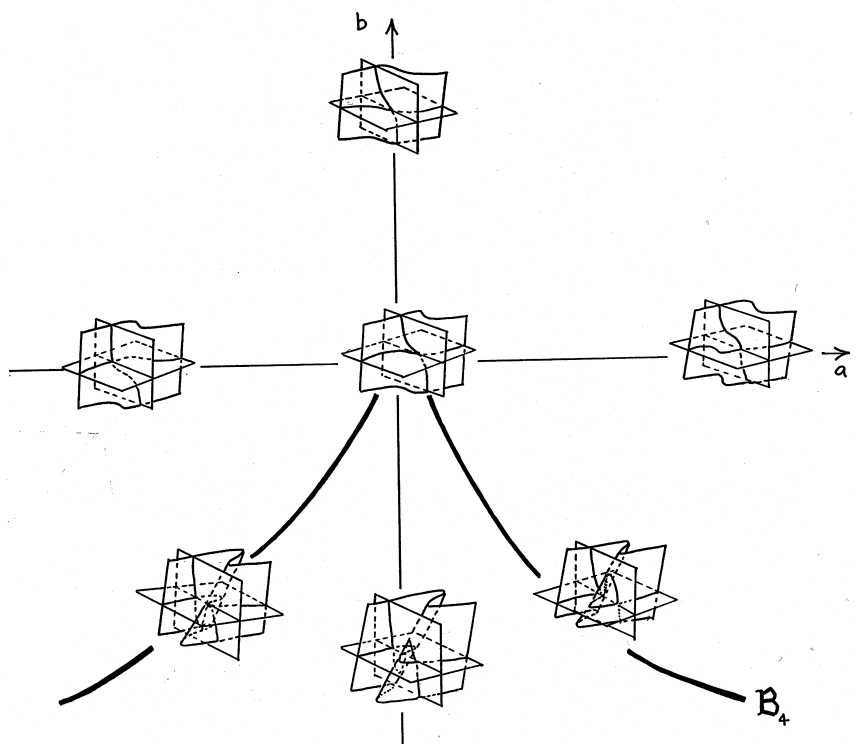

FIG. $4.18 A_{0}^{3} \mid A_{2}$

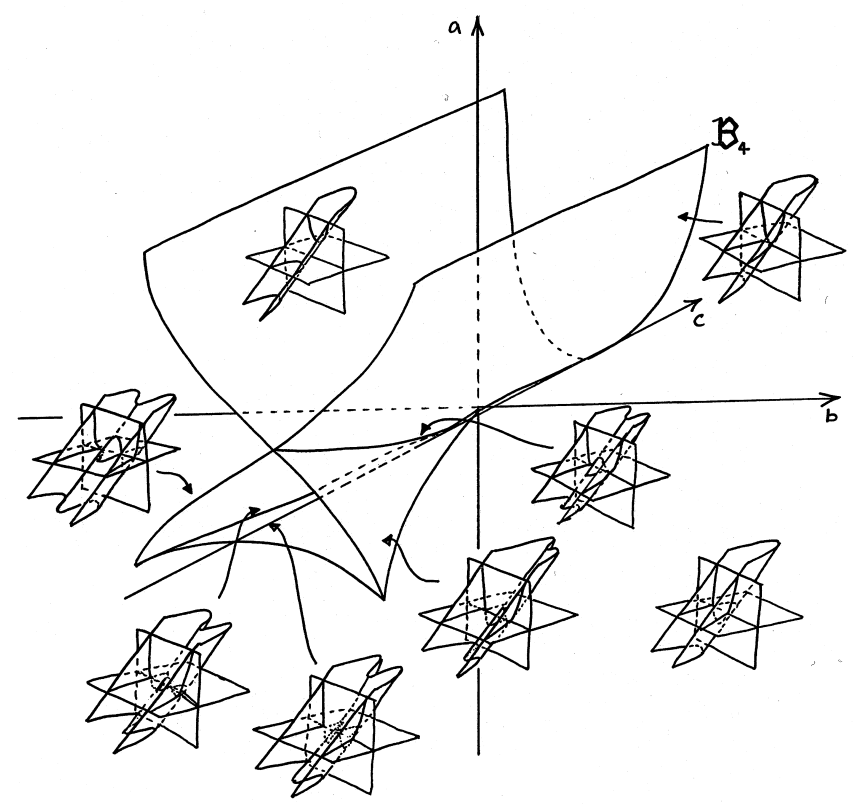

FIG. $4.19 A_{0}^{3} \mid A_{3}$ 
Series $\left(A_{0}^{2} \mid A_{k}^{ \pm}\right)\left(A_{0}\right)$. An $\mathscr{A}_{e}$-versal unfolding is given by $\left(f_{1} ; f_{2} ; f_{3}\right)=$ $\left(x, y, 0 ; X, 0, Y ; \bar{x}, \bar{y}+a, \bar{x}^{2}+p(\bar{y})\right)$ where $p(\bar{y})= \pm \bar{y}^{k+1}+b_{k-1} \bar{y}^{k-1}+$ $\cdots+b_{1} \bar{y}+b_{0}$. The non-empty strata of $\mathscr{B}$ are $\mathscr{B}_{2}$ and $\mathscr{B}_{4}$ which are given by the discriminant of $p(\bar{y})$ and $p(-a)=0$, respectively.

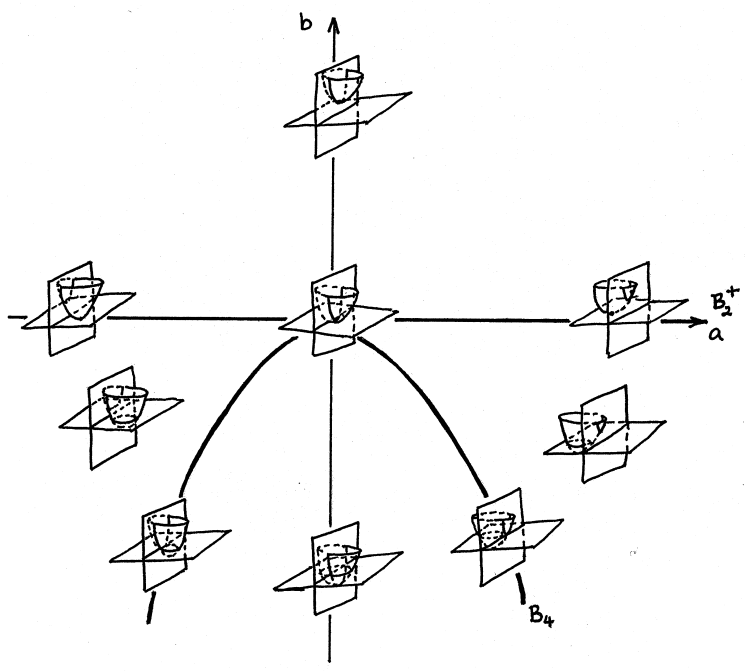

FIG. $4.20\left(A_{0}^{2} \mid A_{1}^{+}\right)\left(A_{0}\right)$

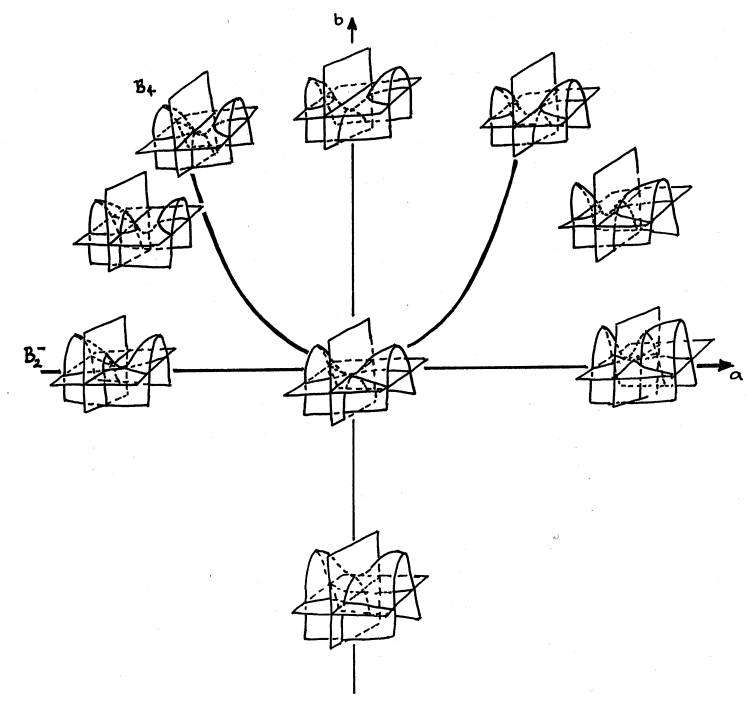

FIG. $4.21\left(A_{0}^{2} \mid A_{1}^{-}\right)\left(A_{0}\right)$ 


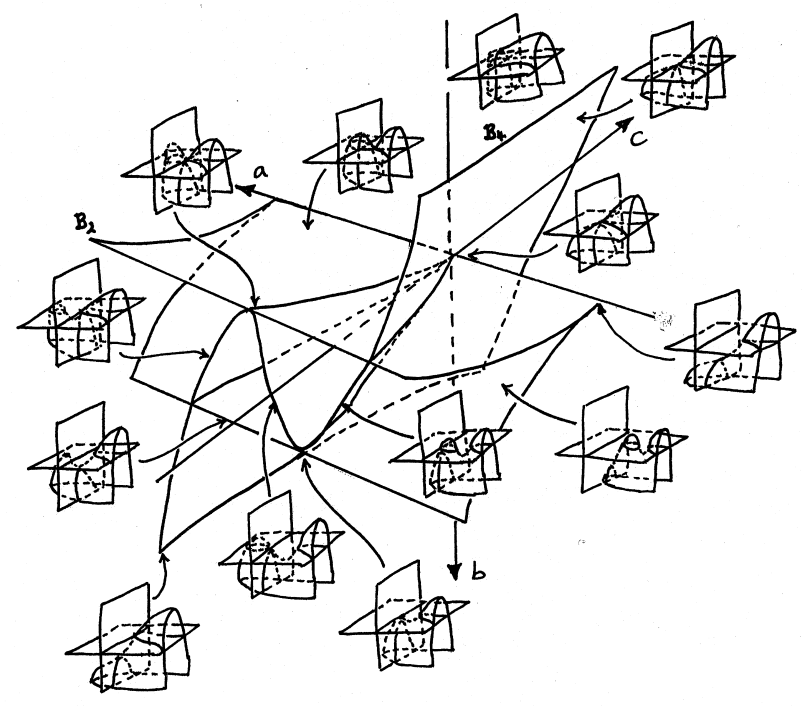

FIG. $4.22\left(A_{0}^{2} \mid A_{2}\right)\left(A_{0}\right)$

Series $\left(A_{0}^{2} \mid A_{\infty}\right)\left(A_{0}\right) \mid A_{k}$. An $\mathscr{A}_{e}$-versal unfolding is given by $\left(f_{1} ; f_{2} ; f_{3}\right)=$ $(x, y, 0 ; X, 0, Y ; \bar{x}, \bar{y}, \bar{x} \bar{y}+p(\bar{x}))$ where $p(\bar{x})=\bar{x}^{k+1}+a_{k} \bar{x}^{k}+\cdots+a_{1} \bar{x}+a_{0}$. The non-empty strata of $\mathscr{B}$ are $\mathscr{B}_{2}$ and $\mathscr{B}_{4}$, given by $a_{0}=0$ and by the discriminant of $p(\bar{x})$, respectively.

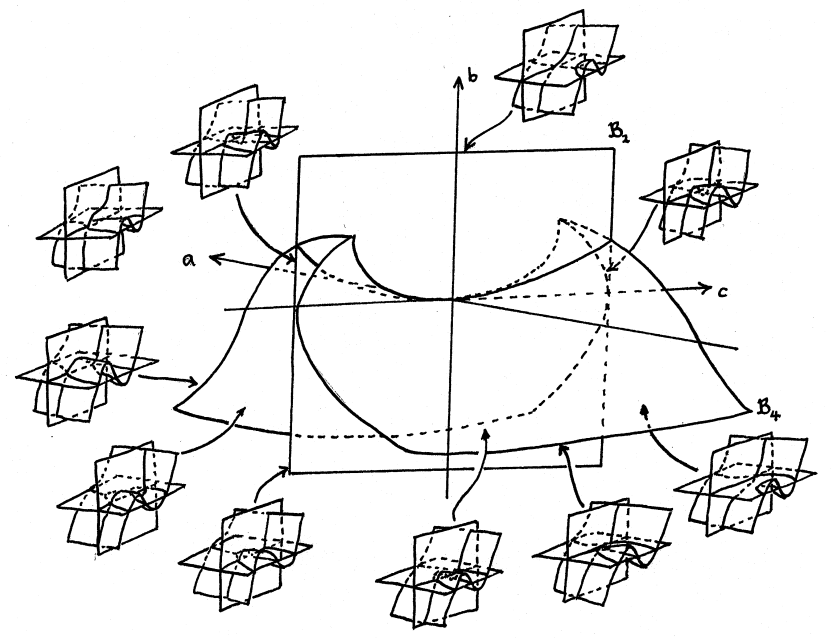

FIG. $4.23\left(A_{0}^{2} \mid A_{\infty}\right)\left(A_{0}\right) \mid A_{2}$ 
We now consider the remaining non-simple trigerms.

Types $A_{0}^{2} S_{0}\left|A_{1}, A_{0}^{2} S_{0}\right| A_{1}(0)^{ \pm}, A_{0}^{2} S_{0} \mid A_{1}(1)^{ \pm}$These trigerms consists of two immersions meeting a cross-cap. For brevity, we will consider all the cases simultaneously. Note that $A_{0}^{2} S_{0} \mid A_{1}$ is a unimodular family (with $a \neq 0,1$ ), the remaining cases arising from the exceptional values of the modulus. In all cases $a, b, c$ denote the unfolding parameters and for $A_{0}^{2} S_{0} \mid A_{1}$ we take the modulus as an unfolding parameter (denoting it by $\bar{a}$ ). An $\mathscr{A}_{e}$-versal unfolding is given by $\left(x, y, 0 ; X, 0, Y ; \bar{y} p(\bar{x}, \bar{y}), \bar{x}, \bar{x}+\bar{y}^{2}+c\right)$ where $p$ defines $D^{2}\left(f_{3}\right)$ and is given by $\bar{x}+\bar{a} \bar{y}^{2}+b, \bar{x} \pm \bar{y}^{4}+a \bar{y}^{2}+b$, and $\bar{x} \pm \bar{y}^{4}+(1+a) \bar{y}^{2}+b$ for each of the respective cases. We summarise the findings.

For $A_{0}^{2} S_{0} \mid A_{1}$ the $\mathscr{B}_{1}$ and $\mathscr{B}_{2}$ strata are empty; $\mathscr{B}_{3}$ has two components which are given by $f_{1}$ meeting a singular point of $f_{3}(b-c=0)$ and likewise for $f_{2}(b=0)$. $\mathscr{B}_{4}$ points are given by the intersection curve of $f_{1}$ and $f_{2}$ meeting $f_{3}$ tangentially, the stratum being given by $c=0$. (The other possible scenarios of $f_{1}$ or $f_{2}$ meeting $D^{2}\left(f_{3}\right)$ tangentially do not occur.) Finally, $\mathscr{B}_{5}$ points occur where $f_{1}$ and $f_{2}$ meet $D^{2}\left(f_{3}\right)$, the condition being $\bar{a} c-b=0$ with $c \leq 0$.

For $A_{0}^{2} S_{0} \mid A_{1}(0)^{ \pm}$the $\mathscr{B}_{4}$ stratum has the extra component $4 b \mp a^{2}$ with $a \leq 0$ ( + case) and $a \geq 0$ ( - case), due to tangency of $f_{2}$ and $D^{2}\left(f_{3}\right)$ in the target; and $\mathscr{B}_{5}$ takes the form $\pm c^{2}-a c+b=0$ with $c \leq 0$.

Similarly, $A_{0}^{2} S_{0} \mid A_{1}(1)^{ \pm}$follows $A_{0}^{2} S_{0} \mid A_{1}$ only $\mathscr{B}_{4}$ has the extra component $\pm a^{2}-4 b+4 c=0$ with $a \leq 0$ ( + case) and $a \geq 0$ ( - case), due to tangency of $f_{1}$ and $D^{2}\left(f_{3}\right)$ in the target; and $\mathscr{B}_{5}$ takes the form $\pm c^{2}-a c+b-c=0$ with $c \leq 0$.

Type $A_{0}^{2} S_{1}^{ \pm} \mid A_{1}$ The trigerm consists of two immersions meeting an $S_{1}^{ \pm}$singularity. An $\mathscr{A}_{e}$-versal unfolding is given by $(x, y, 0 ; X, 0, Y ; \bar{y} p(\bar{x}, \bar{y}), \bar{x}, \bar{x}+$ $\left.\bar{y}^{2}+c\right)$ where $p(\bar{x}, \bar{y})=\bar{y}^{2} \pm \bar{x}^{2}+b \bar{x}+a$ defines $D^{2}\left(f_{3}\right)$. The $\mathscr{B}_{1}$ stratum is defined by $\Delta(p(\bar{x}, 0))$, that is $b^{2} \mp 4 a=0 ; \mathscr{B}_{2}$ is empty. $\mathscr{B}_{3}$ has two components, $\pm c^{2}-b c+a=0$ and $a=0$, which occur for the same geometrical reasons as in case $A_{0}^{2} S_{0} \mid A_{1}$ above. Similarly, $\mathscr{B}_{4}$ and $\mathscr{B}_{5}$ points occur as in case $A_{0}^{2} S_{0} \mid A_{1}$, the strata being given by $c=0$ and $a-c=0$ with $a \leq 0$, respectively.

Type $A_{0}^{2} S_{0} \mid A_{2}$ This trigerm consists of two immersions and a cross-cap meeting with $A_{2}$ contact. An $\mathscr{A}_{e}$-versal unfolding is given by $(x, y, 0 ; X, 0, Y$; $\left.\bar{y}^{2}, \bar{x}, \bar{x}+\bar{x} \bar{y}+\bar{y}^{3}+c \bar{y}^{2}+b \bar{y}+a\right)$ and $p(\bar{x}, \bar{y})=\bar{x}+\bar{y}^{2}+b$ defines $D^{2}\left(f_{3}\right)$. For the same reasons as in case $A_{0}^{2} S_{0} \mid A_{1}$, we find that the $\mathscr{B}_{1}$ and $\mathscr{B}_{2}$ strata are empty, the two components of $\mathscr{B}_{3}$ are $a-b=0$ and $b=0 ; \mathscr{B}_{4}$ is the 'twisted-cuspidal-edge surface' $\Delta\left(\bar{y}^{3}+c \bar{y}^{2}+b \bar{y}+a\right)$; and $\mathscr{B}_{5}$ is given by $a-b c=0$ with $b \leq 0$. 


\subsection{Bifurcation of Higher Multigerms}

Type $A_{0}^{4}$ and Series $\left(A_{0}^{3} \mid A_{k}\right)\left(A_{0}\right)$ An $\mathscr{A}_{e}$-versal unfolding of $\left(A_{0}^{3} \mid A_{k}\right)\left(A_{0}\right)$ is given by $\left(f_{1} ; f_{2} ; f_{3} ; f_{4}\right)=(x, y, 0 ; X, 0, Y ; 0, \bar{x}, \bar{y} ; \bar{X}+a, \bar{Y}, \bar{Y}+p(\bar{X}))$ where $p(\bar{X})=\bar{X}^{k+1}+b_{k-1} \bar{X}^{k-1}+\cdots+b_{1} \bar{X}+b_{0}$. The non-empty strata of $\mathscr{B}$ are $\mathscr{B}_{4}$ and $\mathscr{B}_{5}$ which are given by the discriminant of $p(\bar{X})$ and $p(-a)=0$, respectively. Note that these results also apply to the case $A_{0}^{4}$ (this case could be included as part of the above series but, by definition, we prefer not to).

PRoOF. Fortunately, all of the 4-germs that we must consider have nonsingular branches. This simplifies matters as the $\mathscr{B}_{1}$ and $\mathscr{B}_{3}$ strata are immediately empty, as are all of the $D^{2}$ curves. Now

$$
d f_{4}=\left(\begin{array}{cc}
1 & 0 \\
0 & 1 \\
\partial p / \partial \bar{X} & 1
\end{array}\right)
$$

and clearly no two of the branches can intersect tangentially, so $\mathscr{B}_{2}$ is empty. The intersection curves of each pair of branches $f_{i}, f_{j}$ with $i, j \leq 3$ are the three coordinate axes. Tangency of an axis with $f_{4}$ is a determinant condition involving $d f_{4}$ and one sees that only the u-axis (the intersection curve of $f_{1}$ and $f_{2}$ ) can intersect $f_{4}$ tangentially and this requires that $\partial p / \partial \bar{X}=0$. Intersection requires, in addition, that $\bar{Y}, p(\bar{X})=0$ and $\mathscr{B}_{4}$ is therefore given by the discriminant of $p$. Finally, all four branches intersect, necessarily at the origin in $\mathrm{R}^{3}$, when $p(-a)=0$, and this determines $\mathscr{B}_{5}$.

Type $\left(A_{0}^{2} \mid A_{1}\right)\left(A_{0}^{2}\right)$ An $\mathscr{A}_{e}$-versal unfolding is given by $\left(f_{1} ; f_{2} ; f_{3} ; f_{4}\right)=$ $\left(x, y, 0 ; X, 0, Y ; 0, \bar{x}, \bar{y} ; \bar{X}+d, \bar{Y}, \bar{Y}^{2}+\bar{X} \bar{Y}+\bar{a} \bar{X}^{2}+b \bar{X}+c\right)$. Using similar arguments to those above we find that the bifurcation set for this unimodular family is made up as follows. The $\mathscr{B}_{2}$ stratum is given by $(1-4 \bar{a}) c+b^{2}=0$ due to tangency of the $f_{1}$ and $f_{4}$ sheets. The $\mathscr{B}_{4}$ stratum has two components given by $b^{2}-4 \bar{a} c=0$ and $(4 \bar{a}-1) d^{2}-4 b d+4 c=0$ due to tangency of $f_{4}$ with the intersection curves of $f_{1}, f_{2}$ and $f_{1}, f_{3}$, respectively. Finally $\mathscr{B}_{5}$ is given by $\bar{a} d^{2}-b d+c=0$.

Finally, we deal with the 5-germs. Again, $\mathscr{B}_{1}, \mathscr{B}_{3}$, and all $D^{2}$ curves are empty.

Type $A_{0}^{5}$ The 5-germ consists of 5 planes going through the origin and forms a bimodular family. An $\mathscr{A}_{e}$-versal unfolding is $\left(f_{1} ; f_{2} ; f_{3} ; f_{4} ; f_{5}\right)=$ $(x, y, 0 ; X, 0, Y ; 0, \bar{x}, \bar{y} ; \bar{X}, \bar{Y}, \bar{X}+\bar{Y}+d ; \tilde{x}, \tilde{y}, \bar{a} \tilde{x}+\bar{b} \tilde{y}+c)$. The only nonempty stratum of $\mathscr{B}$ is $\mathscr{B}_{5}$. This has five components given by $(c-d)(\bar{b} d-$ c) $(\bar{a} d-c) c d=0$ corresponding to the intersection of $f_{1}, \ldots, \hat{f}_{i}, \ldots, f_{5}$ where $i=1, \ldots, 5$ (and $\hat{f}_{i}$ denotes the omission of $f_{i}$ from this list), respectively. (Degeneracies of type $\mathscr{B}_{2}$ and $\mathscr{B}_{4}$ occur only for exceptional values of the 
moduli. For example, tangency of $f_{5}$ with the intersection curve of $f_{1}, f_{4}$ occurs when $\bar{a}-\bar{b}=0$ and $\bar{a} d-c=0$. But such points are exceptional points on the $\mathscr{B}_{5}$ component formed by the intersection of $f_{1}, f_{2}, f_{4}, f_{5}$.)

Type $\left(A_{0}^{3} \mid A_{1}\right)\left(A_{0}^{2}\right)$ Again, we have 5 planes going through the origin, only this time the fourth branch meets the intersection curve of the first two branches tangentially. An $\mathscr{A}_{e}$-versal unfolding is given by $\left(f_{1} ; f_{2} ; f_{3} ; f_{4} ; f_{5}\right)=(x, y$, $\left.0 ; X, 0, Y ; 0, \bar{x}, \bar{y} ; \bar{X}, \bar{Y}, \bar{Y}+\bar{X}^{2}+c \bar{X}+d ; \tilde{x}, \tilde{y}, \tilde{x}+\bar{a} \tilde{y}+b\right)$. The only nonempty strata of $\mathscr{B}$ are $\mathscr{B}_{4}$ and $\mathscr{B}_{5}$. We obtain non-transverse triple points from tangencies of $f_{4}$ with the intersection curve of $f_{1}, f_{2}$, and from tangencies of $f_{5}$ with the intersection curves $f_{1}, f_{4}$ and $f_{2}, f_{4}$. The three respective components of $\mathscr{B}_{4}$ are given by

$$
\left(c^{2}-4 d\right)\left((\bar{a} c-1)^{2}-4 \bar{a}(\bar{a} d-b)\right)\left((c-1)^{2}-4(b-d)\right)=0 .
$$

Finally, the five components which go to make up $\mathscr{B}_{5}$ are given by

$$
(b-d)(\bar{a} d-b)\left(b^{2}-b c+d\right) b d=0 .
$$

\section{REFERENCES}

1. Wik-Atique, R., On the classification of multigerms of maps from $\mathbf{C}^{2} \rightarrow \mathbf{C}^{3}$ under $\mathscr{A}$ equivalence, in Real anc Complex Singularities (J. V. Bruce and F. Tari, eds.), Proceedings of the 5th Workshop on Real and Complex Singularities, São Carlos, Brazil, 1998. CRC Res. Notes Math. 412 (2000), 119-133.

2. Bruce, J. W., Kirk, N. P., du Plessis, A. A., Complete transversals and the classification of singularities, Nonlinearity 10 (1997), 253-275.

3. Bruce, J. W., du Plessis, A. A., Wall, C. T. C., Determinacy and unipotency, Invent. Math. 88 (1987), 521-554.

4. Gibson, C. G., Hobbs, C. A., Singularities of general one-dimensional motions of the plane and space, Proc. Roy. Soc. Edinburgh Sect. A 125 (1995), 639-656.

5. Gibson, C. G., Hobbs, C. A., Singularity and bifurcation for general two-dimensional planar motions, New Zealand J. Math. 25 (1996), 141-163.

6. Gibson, C. G., Hobbs, C. A., Marar, W. L., On the bifurcation of general two-dimensional spatial motions, Acta Appl. Math. 47 (1997), 221-242.

7. Goryunov, V. V., Local invariants of mappings of surfaces into three-space, in Arnold-Gelfand Mathematical Seminars, Geometry and Singularity Theory, (V.I. Arnold and I.M. Gelfand, eds.), Birkhäuser, 1996, 223-255.

8. Hobbs, C. A., On kinematic singularities of low dimension, Ph.D. Thesis, University of Liverpool, 1993.

9. Kirk, N. P., Computational aspects of classifying singularities, LMS JCM 3 (2000), 207-228.

10. Mather, J. N., Stability of $C^{\infty}$-mappings III: Finitely determined map-germs, Inst. Hautes Études Sci. Publ. Math. 35 (1968), 127-156.

11. Mather, J. N., Stability of $C^{\infty}$ mappings IV: Classification of stable map-germs by R-algebras, Inst. Hautes Études Sci. Publ. Math. 37 (1970), 223-248.

12. Mond, D. M. Q., On the classification of germs of maps from $\mathbf{R}^{2}$ to $\mathbf{R}^{3}$, Proc. London Math. Soc. (3) 50 (1985), 333-369. 
13. Mond, D. M. Q., Vanishing cycles for complex analytic maps, in Singularity Theory and its Applications, Warwick 1989, (D. Mond and J. Montaldi, eds.), Lecture Notes in Math. 1462, 1991, 221-234.

14. Morris, R. J., The use of computer graphics for solving problems in singularity theory, in Visualization and Mathematics, (H-C. Hege and K. Polthier, eds.), Springer Verlag, Heidelberg, 1997, 53-66.

15. Wall, C. T. C., Finite determinacy of smooth map-germs, Bull. London Math. Soc. 13 (1981), 481-539.

16. Wilson, L. C., Mappings of finite codimension, unpublished notes.

C. A. HOBBS

SCHOOL OF COMPUTING \& MATHEMATICAL SCIENCES

OXFORD BROOKES UNIVERSITY

GIPSY LANE, HEADINGTON

OXFORD. OX3 0BP

U.K
N. P. KIRK

DEPARTMENT OF MATHEMATICAL SCIENCES DIVISION OF PURE MATHEMATICS

UNIVERSITY OF LIVERPOOL, P.O. BOX 147

LIVERPOOL. L69 3BX

U.K. 